\title{
Occupancy-Based Energy Management in Buildings: Final Report to Sponsors
}

Michael D. Sohn ${ }^{1}$, Douglas R. Black ${ }^{1}$, Phillip N. Price ${ }^{1}$ Yiqing Lin ${ }^{2}$, Rohini Brahme ${ }^{2}$, Amit Surana ${ }^{2}$, Satish Narayanan ${ }^{2}$ Alberto Cerpa ${ }^{3}$, Varick Ericson ${ }^{3}$, Ankur Kamthe ${ }^{3}$

${ }^{1}$ Environmental Energy Technologies Division Lawrence Berkeley National Laboratory Indoor Environment Department Berkeley, CA 94720

${ }^{2}$ United Technologies Research Center East Hartford, CT 06108

${ }^{3}$ School of Engineering University of California, Merced Merced, CA 95343

June 2010 


\section{ACKNOWLEDGMENT}

This work was supported by the Assistant Secretary for Energy Efficiency and Renewable Energy, Office of Building Technology, State and Community Programs of the U.S. Department of Energy under Contract No. DE-AC02-05CH11231 and by the California Energy Commission PIER Buildings program through the California Institute for Energy and the Environment (CIEE), and by the United Technologies Research Center.

\section{DISCLAIMER}

This document was prepared as an account of work sponsored by the United States Government. While this document is believed to contain correct information, neither the United States Government nor any agency thereof, nor The Regents of the University of California, nor any of their employees, makes any warranty, express or implied, or assumes any legal responsibility for the accuracy, completeness, or usefulness of any information, apparatus, product, or process disclosed, or represents that its use would not infringe privately owned rights. Reference herein to any specific commercial product, process, or service by its trade name, trademark, manufacturer, or otherwise, does not necessarily constitute or imply its endorsement, recommendation, or favoring by the United States Government or any agency thereof, or The Regents of the University of California. The views and opinions of authors expressed herein do not necessarily state or reflect those of the United States Government or any agency thereof or The Regents of the University of California.

This report was also prepared as a result of work sponsored by the California Energy Commission (Energy Commission) and the University of California (UC). It does not necessarily represent the views of the Energy Commission, UC, their employees, or the State of California. The Energy Commission, the State of California, its employees, and UC make no warranty, express or implied, and assume no legal liability for the information in this report; nor does any party represent that the use of this information will not infringe upon privately owned rights. This report has not been approved or disapproved by the Energy Commission or UC, nor has the Energy Commission or UC passed upon the accuracy or adequacy of the information in this report. 


\section{Table of Contents}

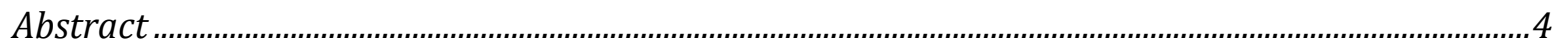

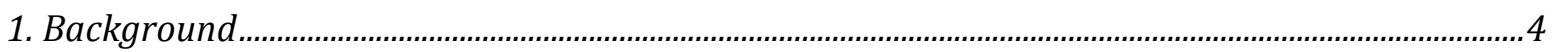

2. Description of Phase 1 Tasks ..........................................................................................................

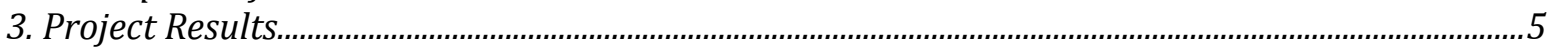

Task 1: Describe Energy Usage in the COB (LBNL Lead Author) ...........................................................5

Task 2: Develop Energy Model of the COB (LBNL Lead Author) ........................................................6

Task 3: Deploy Sensor Network in the S\&E (UC Merced Lead Author).................................................22

Task 4: Develop Energy Controls for the COB (UTRC Lead Author) ....................................................25

Task 5: Develop People Dynamics Model for Building Occupants (UTRC Lead Author).....................32

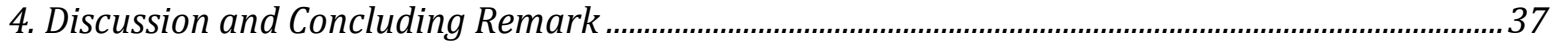

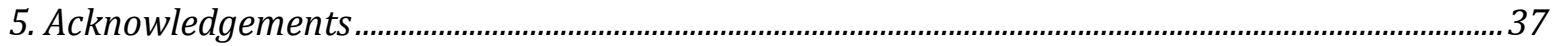

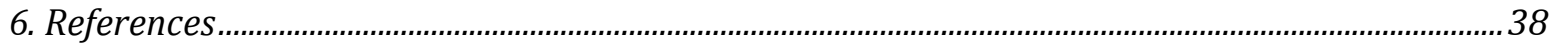

Appendix: Publications Resulting from Research …............................................................................ 40 


\begin{abstract}
The Lawrence Berkeley National Laboratory (LBNL), the University of California Merced (UCM), and the United Technologies Research Center (UTRC) conducted field studies and modeling analyses in the Classroom and Office Building (COB) and the Science and Engineering Building (S\&E) at the University of California, Merced. In the first year, of a planned multiyear project, our goal was to study the feasibility and efficacy of occupancy-based energy management. The first-year research goals were twofold. The first was to explore the likely energy savings if we know the number and location of building occupants in a typical commercial building. The second was to model and estimate people movement in a building. Our findings suggest that a $10-14 \%$ reduction in HVAC energy consumption is possible over typical HVAC operating conditions when we know occupancy throughout the building. With the conclusion of the first-year tasks, we plan to review these results further before this group pursues follow-on funding.
\end{abstract}

\title{
1. Background
}

Heating, ventilating, and air conditioning (HVAC) systems account for $50 \%$ of the total energy budget in buildings (Payne, 1984). Prior research has shown that energy savings are achievable by regulating fresh air ventilation based on the total number of occupants in a building. This procedure, called demand-controlled ventilation, allows 10 to $15 \%$ reduction of HVAC energy compared to setting ventilation rates by assuming maximum occupancy (see e.g., Brandemuehl and Braun, 1999). In many buildings, some rooms are empty during at least part of the day, so there appears the possibility that occupancy-based HVAC controls - supplying HVAC only to occupied rooms - may further reduce energy costs.

Reliable spatially resolved occupancy data are not available in most buildings. Many modern buildings include motion detectors, and temperature and $\mathrm{CO}_{2}$ sensors for light and air flow management, but these sensors present some limitations. Motion detectors do not indicate the number of people present, and temperature and $\mathrm{CO}_{2}$ sensors provide indirect measures that lag in time. If occupancy-based energy management is feasible, a faster distributed sensor network may be needed to give accurate and rapid occupancy estimates or measurements.

The lack of reliable data to estimate energy savings potential, or to test the feasibility of determining local-scale occupancy, is a clear roadblock to further exploration. We therefore proposed to investigate occupancy-based energy control strategies on two simultaneous fronts. One is to benchmark the energy savings potential of occupancybased HVAC controls for various levels of occupancy knowledge. The second is to determine the level of occupancy knowledge obtainable. For the first, we developed models of energy consumption and control strategies. For the second, we installed a 40-node "smart camera" network in a portion of the Science and Engineering Laboratory 
(S\&E) building at UC Merced and developed coarse-scale models of occupancy dynamics.

This project was planned in three phases:

Phase 1: Feasibility and efficacy study (12 months)

Phase 2: Refine hardware and software tools, and link with other DOE programs (12 months)

Phase 3: Prototype implementation and testing (12 months)

We report on our findings from Phase 1 . We are reviewing our results, and have not planned follow-up proposals for Phases 2 and 3.

\section{Description of Phase 1 Tasks}

The purpose of Phase 1 was to conduct various order-of-magnitude calculations to estimate the feasibility of occupancy-based estimation and the potential energy savings. We also conducted research to determine technology limits. In this report, we summarize our work on five tasks:

1. Describe energy usage in the UC Merced Classroom and Office Building (COB);

2. Develop an energy model of the COB;

3. Deploy a sensor network in the S\&E;

4. Develop energy controls for the COB; and

5. Develop a people dynamics model for occupants in the S\&E.

We describe our results for each task in the following section.

\section{Project Results}

\section{Task 1: Describe Energy Usage in the COB (LBNL Lead Author)}

This task gathered data to support this project, and a complementary project for the DOE and the California Energy Commission titled "Real-Time Visualization of Energy Performance in Buildings (Piette et al., 2010)." Data include 15-minute electric power for the building as a whole and for four specific circuits; heating and cooling power provided in the form of hot and chilled water from a "district" system that serves the entire campus; HVAC fan energy; and indoor temperature at every thermostat. Additionally, hourly weather data were compiled using a combination of sources, as discussed below. The dashed lines in figures T2-5 through T2-12 show examples of some of the building data. 


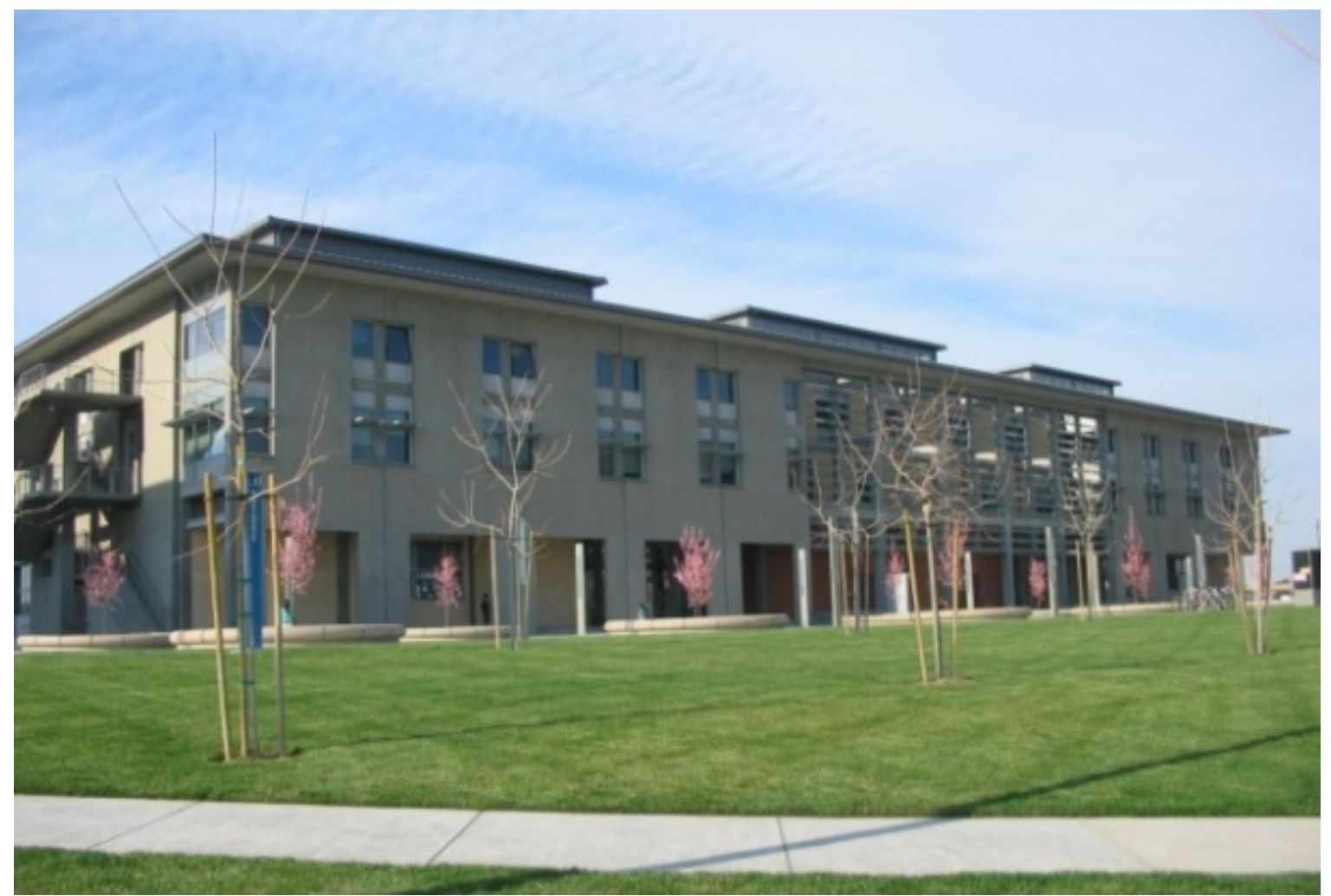

Figure T1-1: The classroom and office building at UC Merced.

\section{Task 2: Develop Energy Model of the COB (LBNL Lead Author)}

We developed an energy model of the UC Merced COB using data from Task 1 and EnergyPlus version 3.1. The building model will support future energy analyses of the $\mathrm{COB}$, and tasks for a complementary project for the DOE and the California Energy Commission called "Real-Time Visualization of Energy Performance in Buildings (Piette et al., 2010)." A description of the building, the model, and efforts to calibrate the model to data follow.

\section{Model Description}

Figure T2-1 shows a model representation of the COB. The building shell is tilt-up concrete panels or curtain-wall frame construction. The concrete panels consist of 12 inches of concrete, interior insulation and a layer of gypsum board. The curtain walls consist of steel siding, exterior gypsum board, insulation, and interior gypsum board. Nearly all interior walls consist of gypsum board sandwiching a sound attenuation blanket. Some interior walls have two layers of gypsum board on one or both sides. Windows consist of an outside layer of LowEnergy Tint $6 \mathrm{~mm}$, a $13 \mathrm{~mm}$ air gap, and an inside layer of Clear $6 \mathrm{~mm}$. Parameters for each layer are available in the materials 
library in EnergyPlus. The overhangs are three feet deep and made of aluminum grating. We depicted them in EnergyPlus as equivalent two-foot solid overhangs.

Figures T2-2 through T2-4 show the building floorplans. Approximately one-third of the building consists of office space, and the remaining is classrooms. The building model consists of 86 conditioned zones $(13,48$, and 25 zones for the first, second, and third floors, respectively). Fifty-five of the zones represent a physical room in the building and the remaining zones represent multiple, adjacent, rooms operating under similar energy usage/requirements. Each model zone includes an "internal mass" that represents the thermal storage capacity of the room(s) (e.g., interior walls, furnishings, paper, books, etc.).

Cooling and heating energy are delivered from campus chilled water and boiler plants. The building HVAC is a dual-fan, dual-duct, variable air-volume system with an economizer. Dual-fan refers to separate cooling fans (two 35,000 CFM fans in parallel) and heating fan (one 25,000 CFM fan). The two cooling fans are represented in EnergyPlus as a single 75,000 CFM fan in EnergyPlus. Dual-duct VAV mixing boxes supplies the room zone.

Nighttime air infiltration is 0.2 air changes per hour. Daytime air infiltration is negligible because ventilation/conditioning pressurizes the building. All classrooms, conference rooms, and auditorium have $\mathrm{CO}_{2}$ sensors for outside air (OA) control (Figures T2-2, T23, and T2-4).

Weather data for 2008 and 2009 consisted of hourly dry-bulb temperature, measured on the roof of the $\mathrm{COB}$, wind speed and direction, recorded at the campus central plant, direct normal radiation, measured 30 miles southwest of the campus at the California Department of Water San Luis Reservoir, and relative humidity, measured at the Fresno International Airport for 2008 and at the Merced Municipal Airport for 2009.

\section{Building Loads}

The primary internal requirements are people, lighting, and plug-load equipment. The Classroom zones have three load schedules to reflect typical use patterns on (1) Mondays, Wednesdays, and Fridays; (2) Tuesdays and Thursdays, and (3) weekends and holidays. Office and all other zones have two load schedules to depict usage on weekdays, and weekends and holidays. Schedules were initially set according to data on building occupancy and operation from John Elliott, the campus energy manager. We later adjusted the schedules to calibrate the model to data.

\section{Model Calibration}

Fifteen-minute sub-meter energy loads have been recorded for several years. The availability of such high temporal resolution meant that we had the opportunity to 
calibrate the model at a substantially greater standard than typically reported in the literature. The data consisted of:

- Whole building cooling and heating power, derived from the flow rate and temperature change in cold and hot water from the district cooling and heating plant.

- HVAC fan power.

- Four energy sub-meters: (1) 4HA - lighting for sections of the 1st, 2nd, and 3rd floors and the exterior, (2) $4 \mathrm{HB}$ - lighting for other sections of the 1st, 2nd, and 3rd floors, (3) 4LA - plug loads for sections of the 2nd and 3rd floor offices, and (4) 4LB - some auditorium lighting and some receptacles in the 1st, 2nd, and 3rd floor classrooms, and 3rd floor offices.

- Internal temperatures.

We predicted electrical loads for lighting, equipment, and fans at 15-minute intervals and compared them to the data. We calibrated electric load by adjusting lighting and equipment schedules. Model parameters were also adjusted to improve the prediction of indoor temperatures. Many parameters affect indoor temperature predictions: the thermal mass of the building and its contents, the heat generated by occupants (each person generates over $100 \mathrm{~W}$ ), the thermal conductivity of the exterior walls, and so on.

Figures T2-5 through T2-10 show model predictions and measured data for electric loads for the whole building, HVAC fans alone, and sub-meters 4HB and 4LB for a winter week (Feb 1-7, 2009) and a summer week (Sep 6-12, 2009). There is good agreement between predictions and measurements for whole building electric demand during occupied periods, but further adjustments to lighting and equipment load schedules are needed to improve agreement during unoccupied periods.

There is good agreement for HVAC fans, with the exception of early morning during cooling season (Sep) and during weekend days. There is somewhat poor agreement for the primary lighting sub-meters 4HA (not shown) and 4HB during occupied periods indicating that more lights are used during the day than are scheduled in the model. There is very good agreement for the primary receptacle sub-meters 4LA (not shown) and 4LB. We believe the 4HA submetering problems are due to model misspecification: the model assumes that the equipment and lights that are served by this circuit are correctly identified in the building blueprints, but it seems increasingly likely that this is not the case. It is not unusual for plans to differ from actual practice --- even "as-built" plans that supposedly indicate the way the building was assembled.

The model also predicts total heating and cooling energy transferred from the heating and cooling coils, respectively. Model predictions were compared to actual heating and cooling energy (from Task 1), which was calculated from logged measurements of supply and return water temperatures and water loop flow rates for both heated and chilled water:

$$
E=0.147 \times Q \times \Delta T
$$


where $\mathrm{E}$ represents energy transferred in $\mathrm{kW}, \mathrm{Q}$ represents water loop flow rate in $\mathrm{gal} / \mathrm{min}$, and delta $\mathrm{T}$ represents the absolute difference between the supply and return water temperatures.

There is poor agreement for heating energy transfer with some under-prediction in winter (Figure T2-9) and less significant over-prediction in summer (Figure T2-10). Cooling energy is greatly over-predicted in winter, somewhat over-predicted during summer weekdays and under-predicted for summer weekend days. Internal loads such as lights, equipment plugged into receptacles, and people affect heating and cooling energy. It appears that, since heating is under-predicted and cooling is over-predicted, internal loads are over-predicted by the model. However, electric loads are fairly well predicted, and even under-predicted in the case of lighting. The number of people in the model has been reduced, but that did not result in significantly improved predictions of heating and cooling energy. Further investigation is needed of the relationship of predicted heating and cooling energy and the model's HVAC control configuration and thermal parameters such as those related to internal mass and exterior surfaces. We are now using newly developed software to adjust model parameters so as to better fit the data, and we hope that this procedure will result in a model that more accurately predicts heating and cooling energy use. However, the improved model is not yet available as the present report is being written.

Model predictions of zone temperatures agree fairly well for summer and less well for winter (Figures T2-11 and T2-12). Zone temperatures decrease and increase during unoccupied periods in the real building much more in the winter and to a lesser extent in the summer as compared to model predictions. Also, the rate at which zone temperatures increase at the beginning of an unoccupied period in summer is greatly over-predicted by the model. Thermal properties of internal mass and exterior surfaces need to be adjusted to improve zone temperature predictions. We believe the automated model tuning discussed above will resolve most of these problems.

Overall, model performance is fairly good, but further improvements in thermal predictions such as zone temperatures and heating and cooling energy are needed. Work in this area is focusing on the model's estimates of thermal mass, the heat transfer characteristics of the building's envelope, and the configuration and control of the HVAC system. 
Table T2-1: Building configuration and areas

\begin{tabular}{|l|c|c|c|c|}
\hline & $\begin{array}{c}\text { Total Building } \\
\text { area }\end{array}$ & $\begin{array}{c}\text { Office } \\
\text { area }\end{array}$ & $\begin{array}{c}\text { Classroom/Conf/Aud } \\
\text { area }\end{array}$ & $\begin{array}{c}\mathrm{CO}_{2} \\
\text { sensors }\end{array}$ \\
\cline { 2 - 5 } $\mathrm{ft}^{2}$ & $\mathrm{ft}^{2}$ & $\mathrm{ft}^{2}$ & Number \\
\hline 1st Floor & 33833 & - & 33833 & 11 \\
\hline 2nd Floor & 32350 & 6600 & 25750 & 25 \\
\hline Total Floor & 27423 & 25932 & 1491 & 3 \\
\hline \% Area & 93606 & 32532 & 61074 & 39 \\
\hline
\end{tabular}




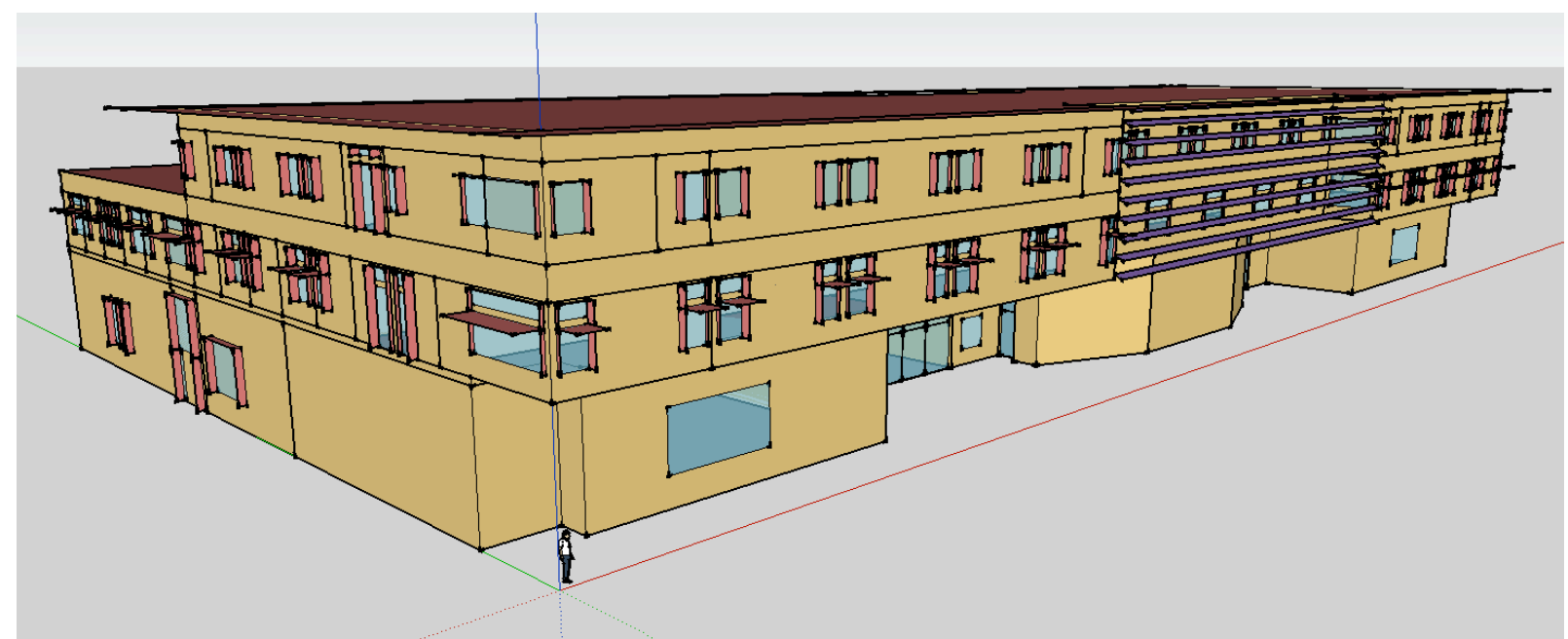

Figure T2-1: Model representation of the COB. 


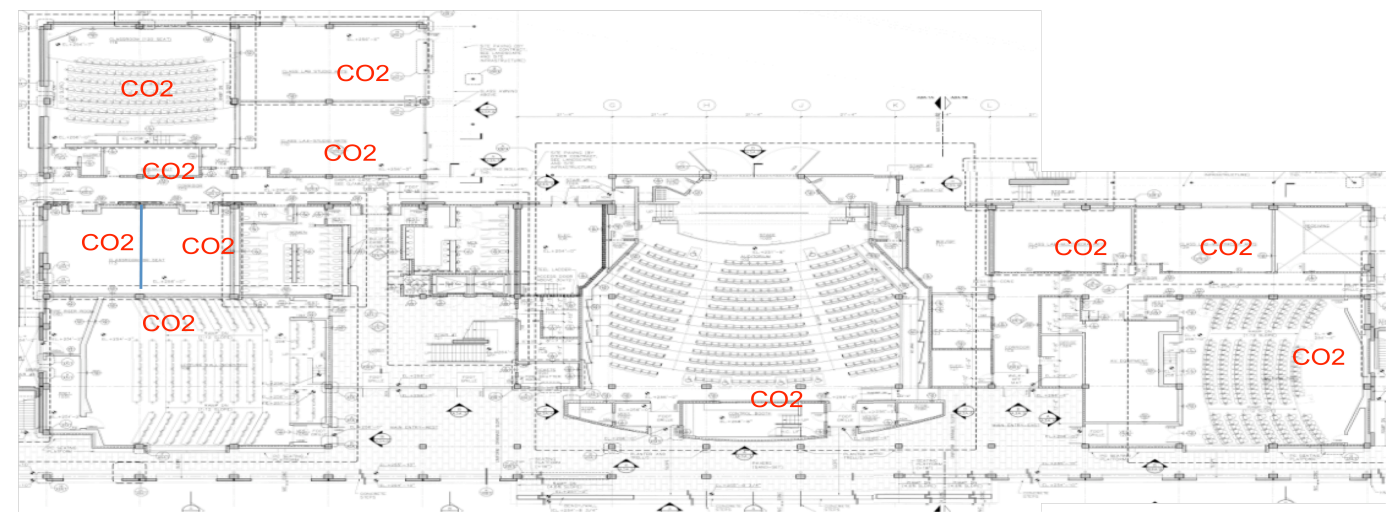

Figure T2-2: Floor plan of 1st floor, and locations of $\mathrm{CO}_{2}$ sensors.

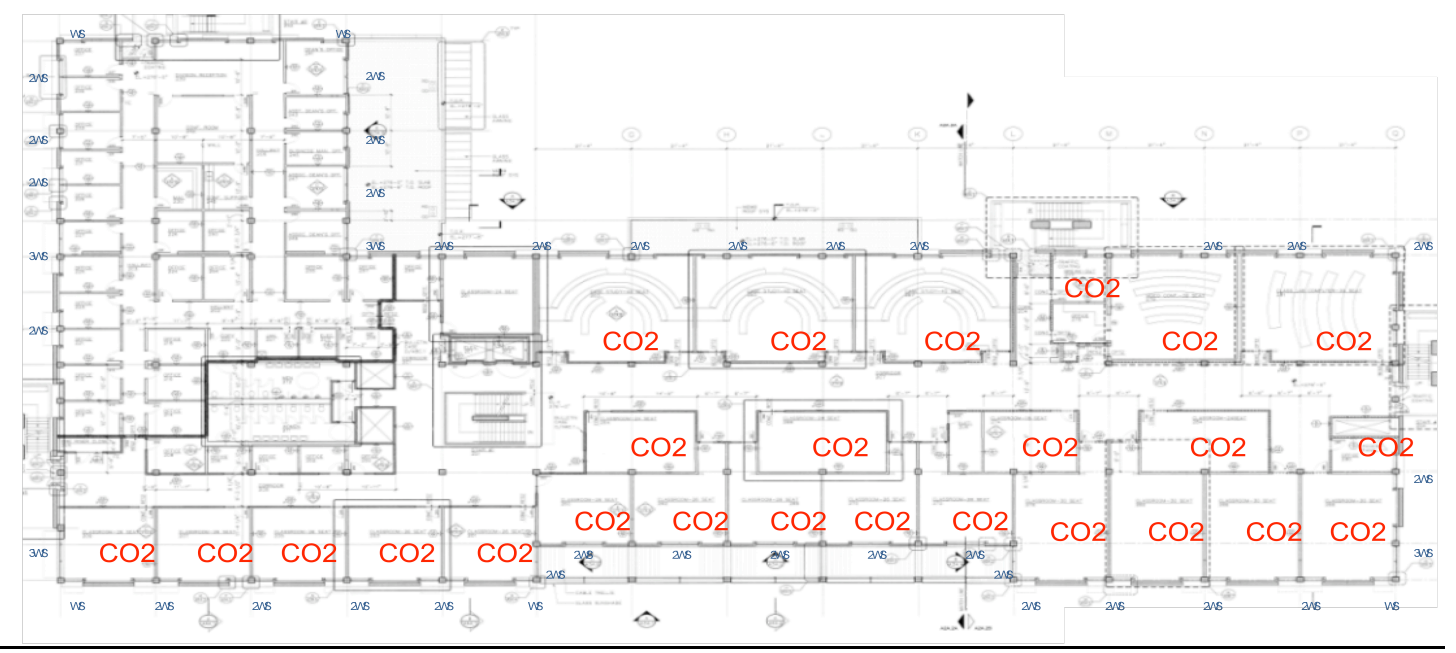

Figure T2-3: Floor plan of 2nd floor, and locations of $\mathrm{CO}_{2}$ sensors. 


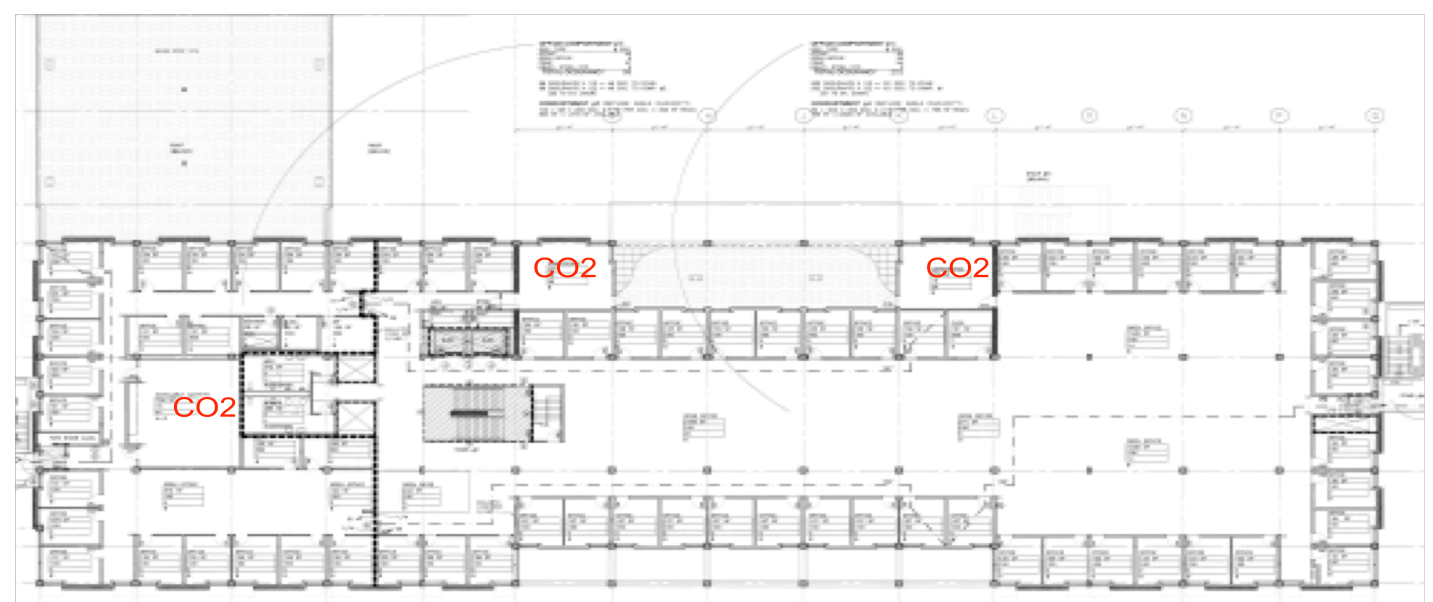

Figure T2-4: Floor plan of 3rd floor, and locations of $\mathrm{CO}_{2}$ sensors. 

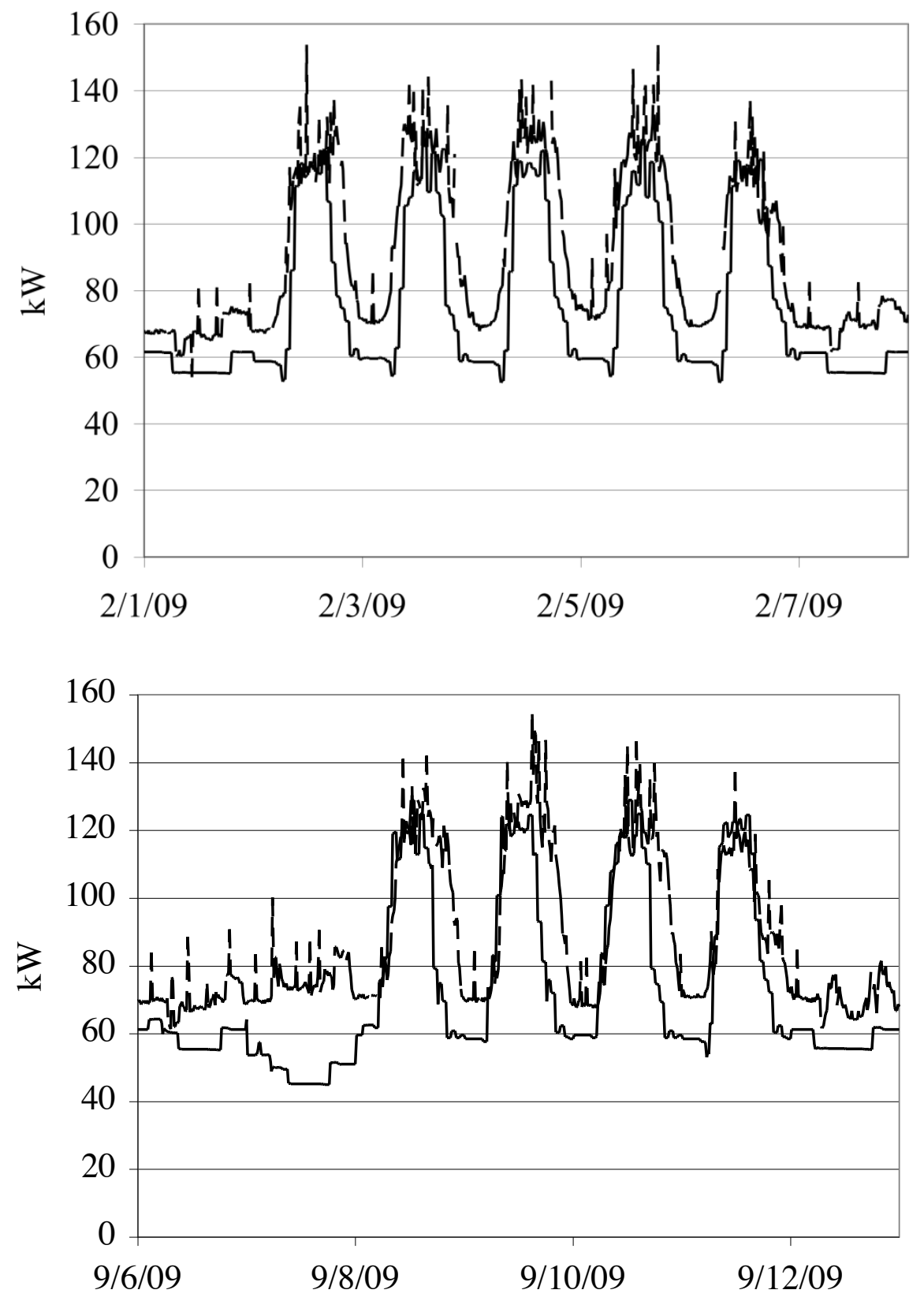

Figure T2-5: Model predictions (solid) compared to measurements (dashed) for wholebuilding loads for a winter (upper) and summer (lower) week. 

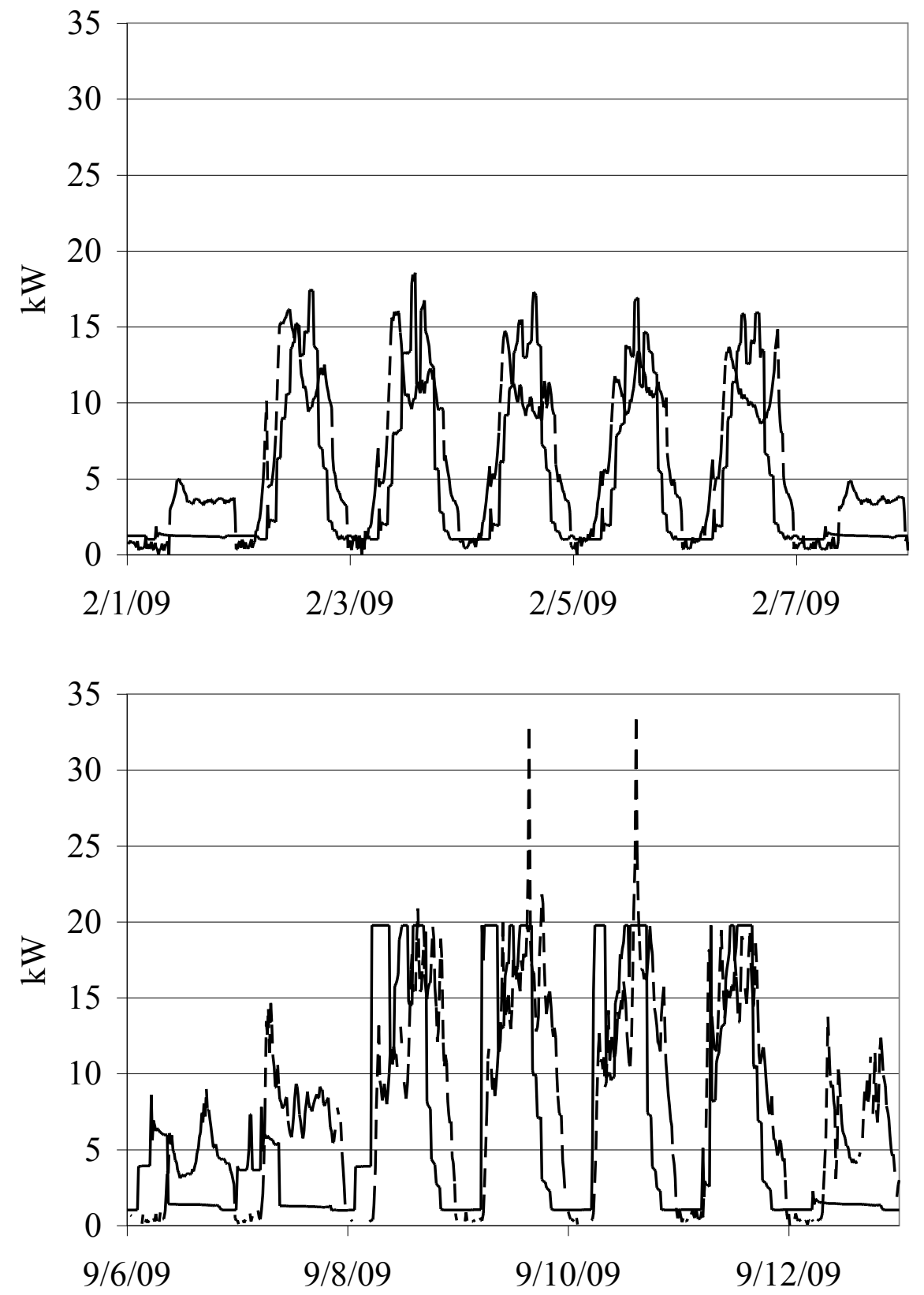

Figure T2-6: Model predictions (solid) compared to measurements (dashed) for HVAC fan loads for the HVAC fans for a winter (upper) and summer (lower) week. 

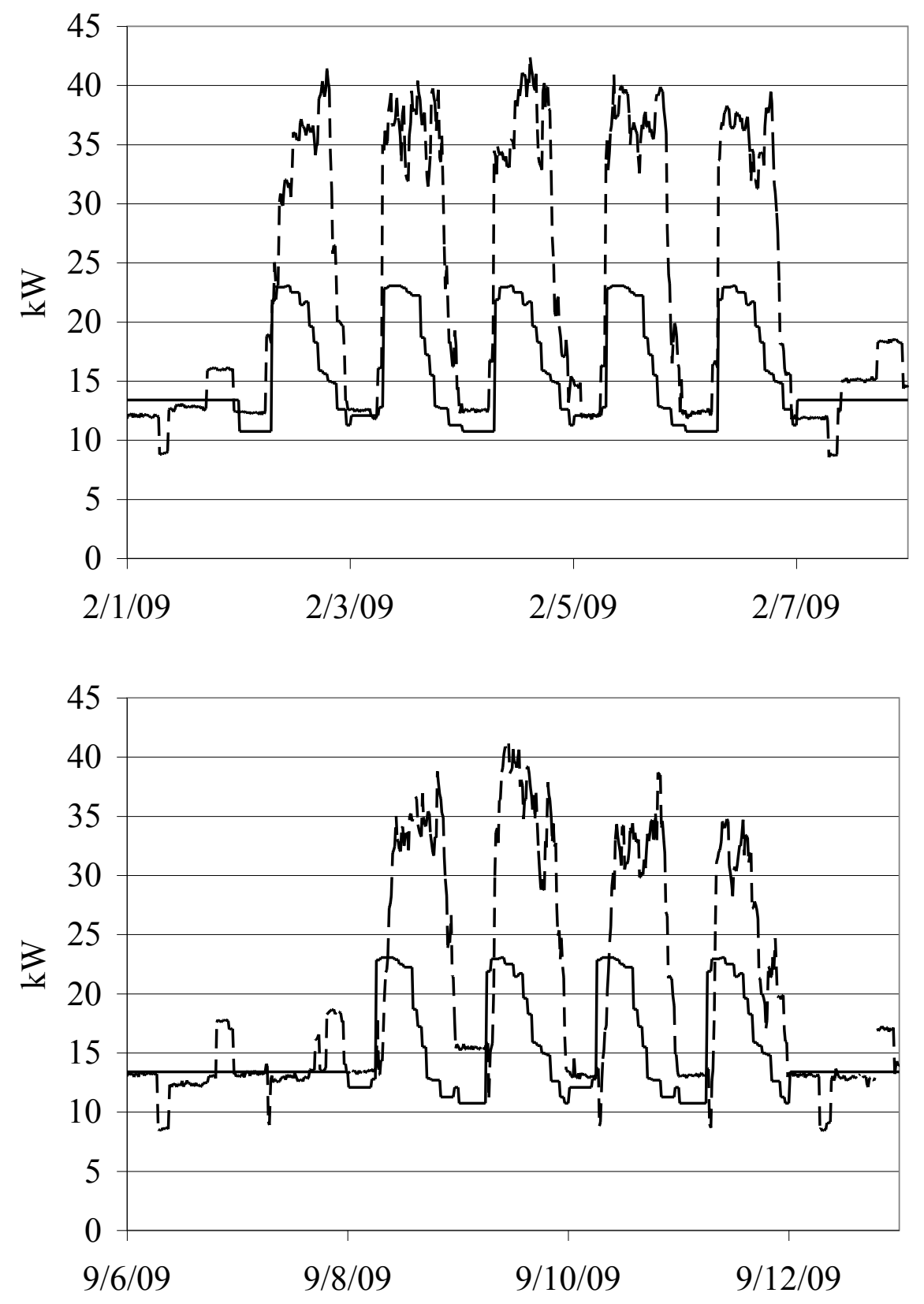

Figure T2-7: Model predictions (solid) compared to measurements (dashed) for submeter 4HB for a winter (upper) and summer (lower) week. 

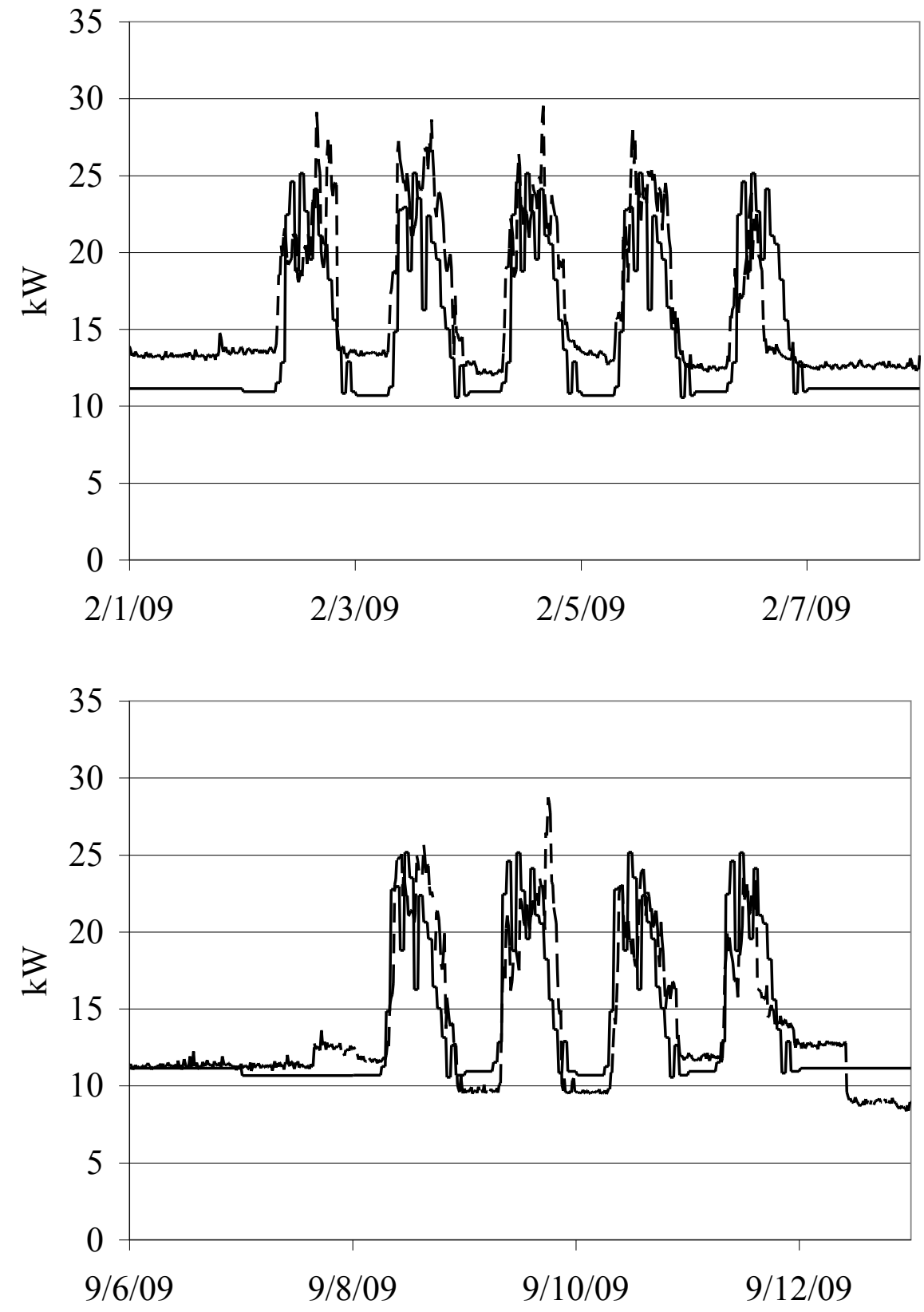

Figure T2-8: Model predictions (solid) compared to measurements (dashed) for submeter 4LB for a winter (upper) and summer (lower) week. 

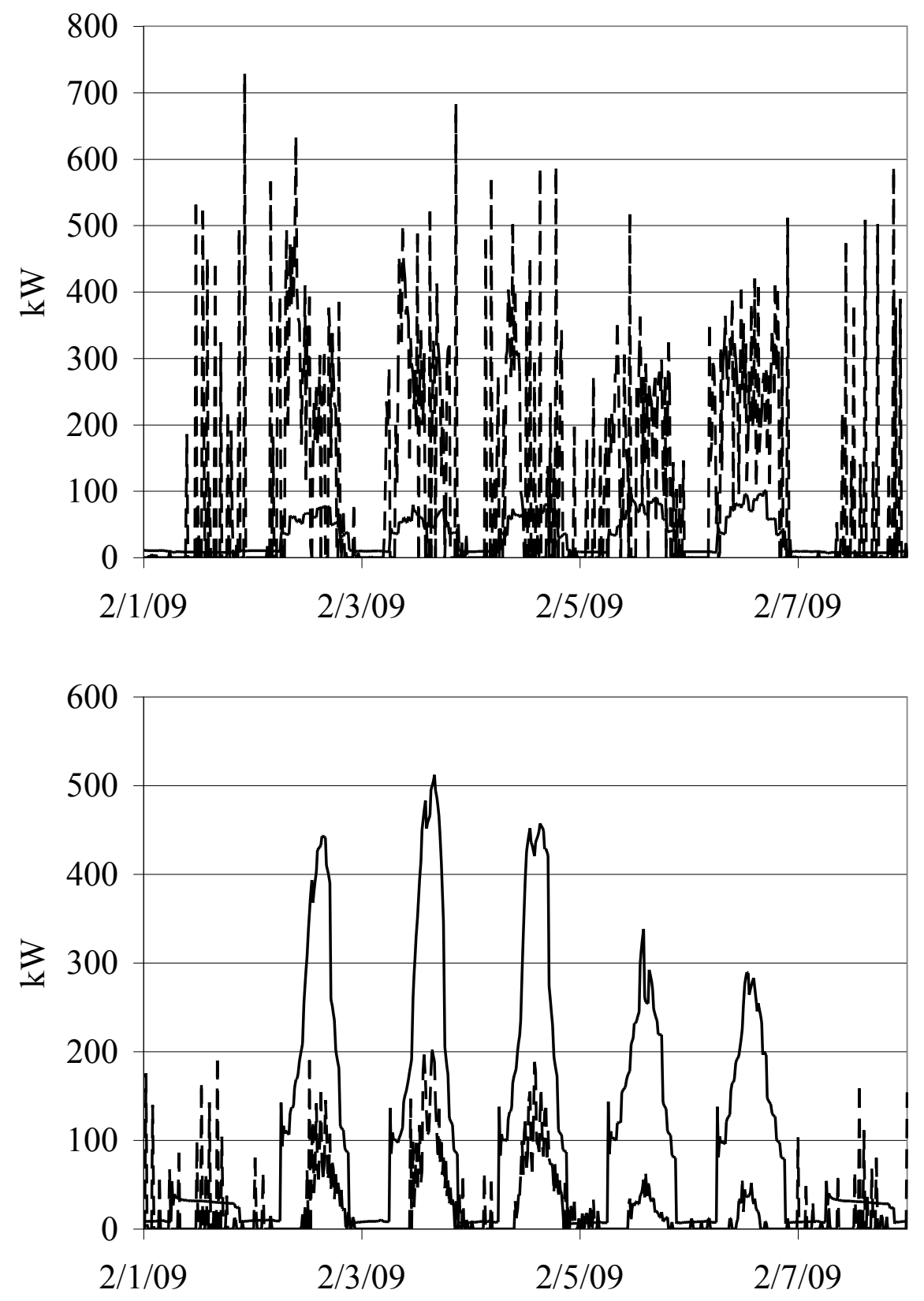

Figure T2-9: Model predictions (solid) compared to measurements (dashed) for heating (upper) and cooling (lower) in February. 

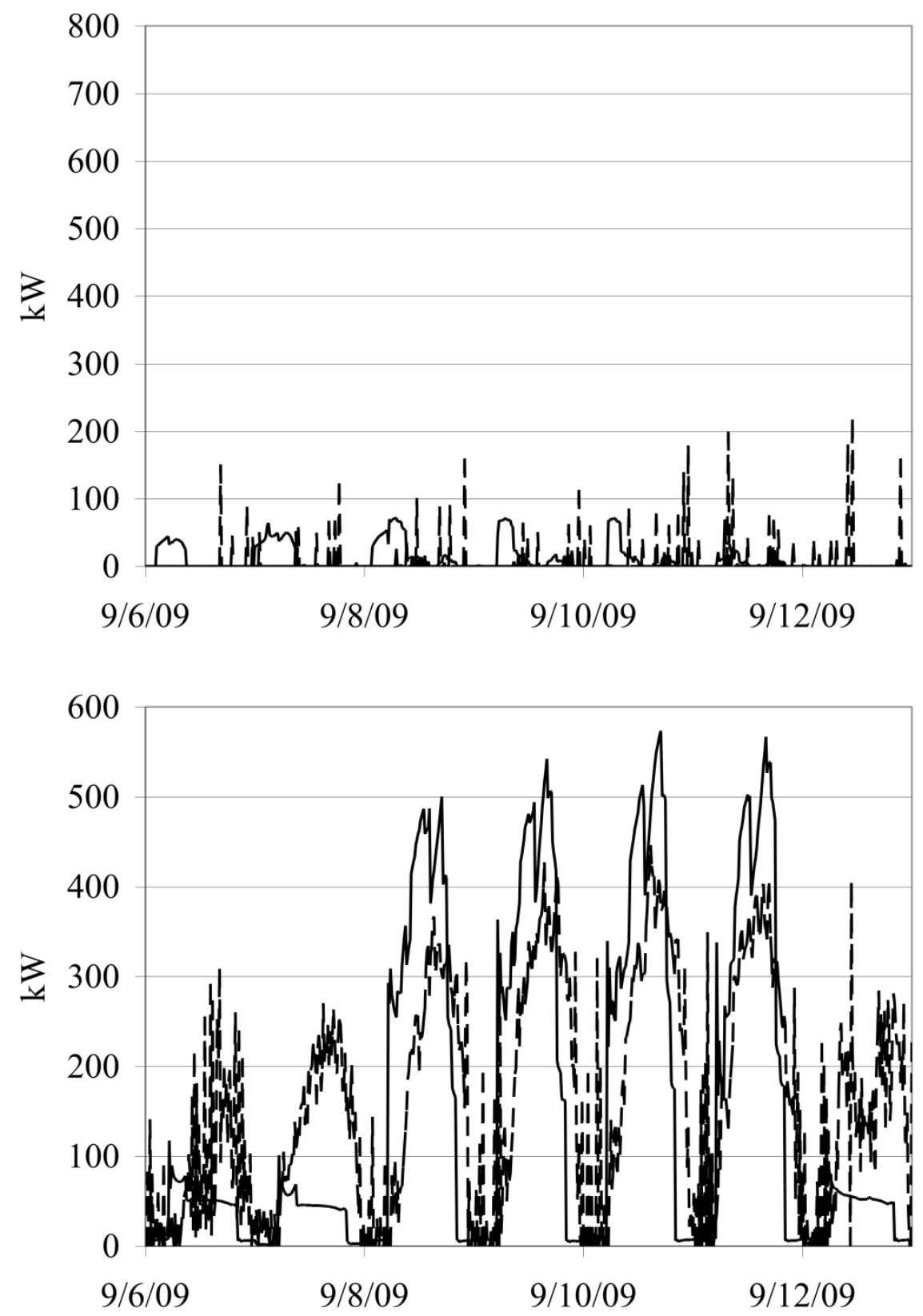

Figure T2-10: Model predictions (solid) compared to measurements (dashed) for heating (upper) and cooling (lower) in September. 

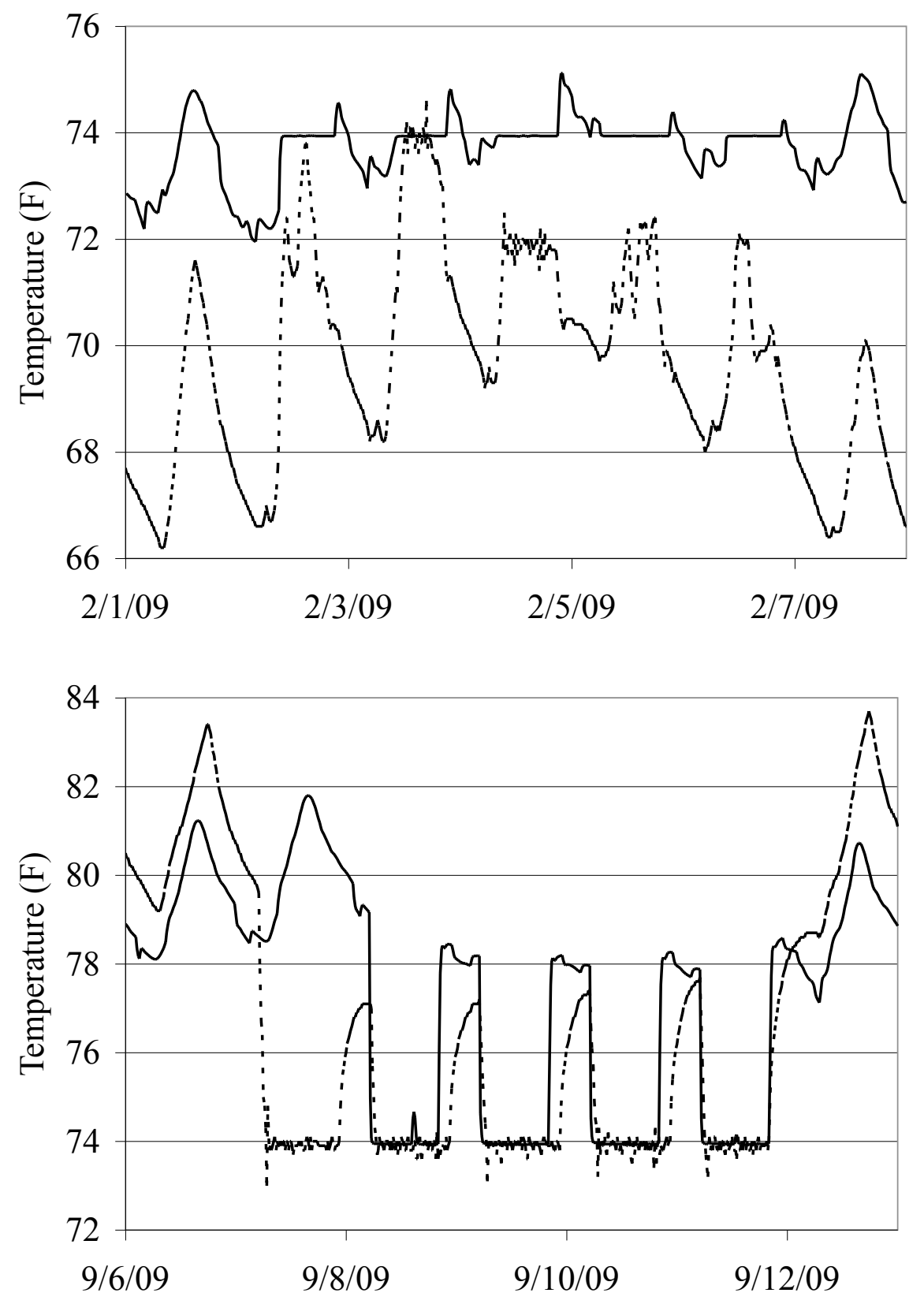

Figure T2-11: Model predictions (solid) compared to measurements (dashed) of zone temperatures for Classroom 209 for Feb 1-7, 2009 and Sep 6-12, 2009. 

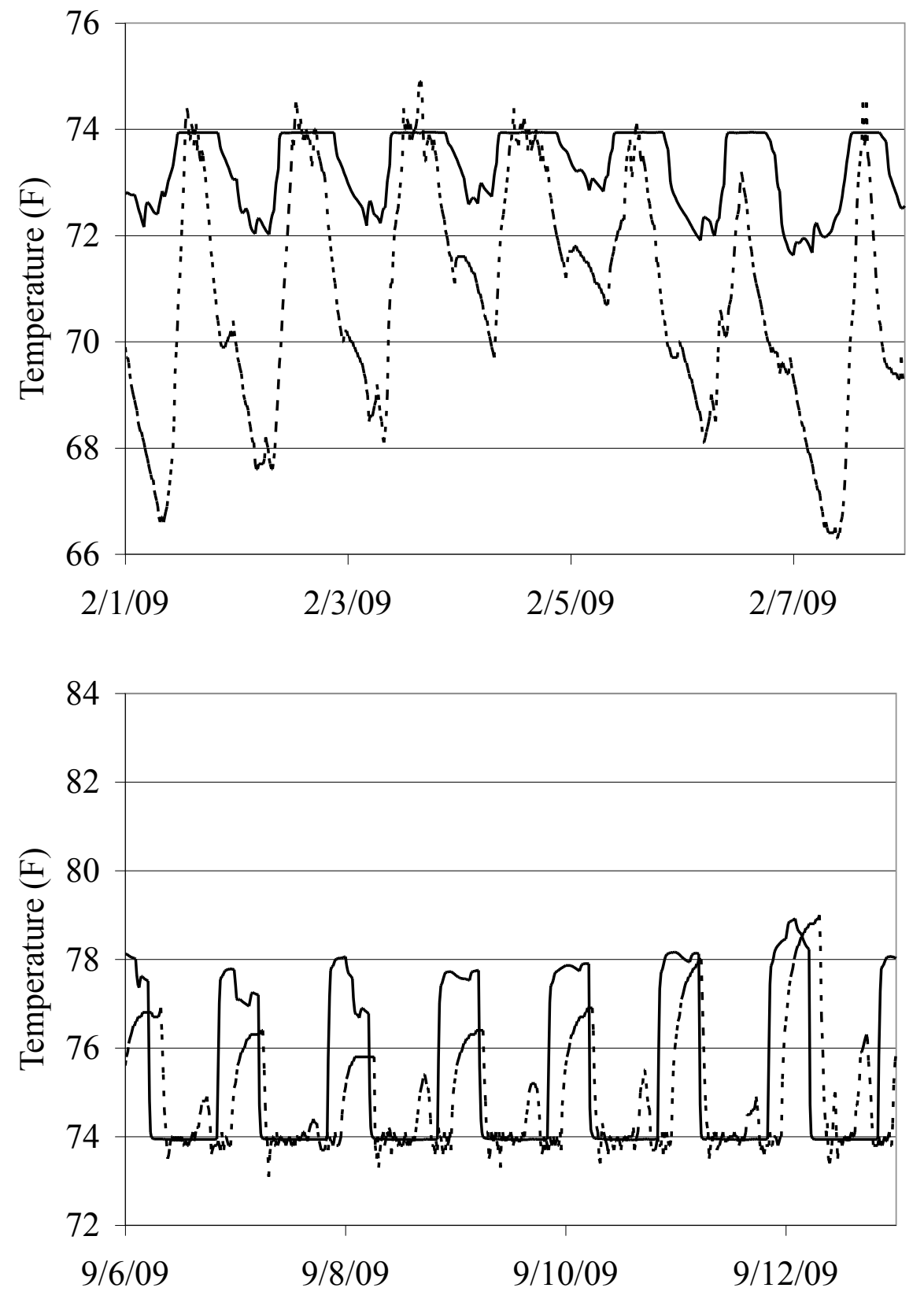

Figure T2-12: Model predictions (solid) compared to measurements (dashed) of zone temperature for Office 317 for Feb 1-7, 2009 and Sep 6-12, 2009. 


\section{Task 3: Deploy Sensor Network in the S\&E (UC Merced Lead Author)}

In this section, we describe the equipment and experiments conducted with the Smart Cameras Object Position Estimation System (SCOPES) (Kamthe et al., 2009), which is a wireless camera sensor network for gathering traces of human mobility patterns in buildings.

The SCOPES system for the S\&E is comprised of 16 sensor nodes on the ceiling of the corridors. The nodes were deployed in groups of three at transition points of the main floor. Each sensor node is comprised of a Cyclops camera interfaced with a Moteiv Tmote Sky module via an intermediate adapter board. The Cyclops is a very low power camera with an on-board 4MHz ATmega128L micro-controller (MCU) and 512KB of external SRAM. Due to the limitation of the total addressable memory, the external SRAM is divided into eight, 64KB memory banks. The Cyclops captures $1064 \times 64$ pixel grayscale images per bank (i.e., 80 total). In each group, multiple nodes sense the same area with coordination to minimize overlap between the image capture periods of the cameras. Whenever a person crosses any one of these transition points, the cameras capture and process the image data.

We execute lightweight processing algorithms on the Cyclops before data transmission to reduce computational-power use. The object detection algorithm running on the Cyclops determines the presence of an object in the image foreground, if any, and updates the background. We developed a modified background subtraction algorithm that labels the pixels in the captured images as an object, a shadow, or background depending upon a preset threshold. Next, we use a connected components algorithm to merge all pixels labeled as an object into a "blob". For each blob, information regarding the centroid ( $x$ and $y$ coordinates) and the number of pixels is recorded. We track the movement of the blob across images. After processing all the images in the current bank, an array of data structures containing information on a maximum of four objects per bank is transferred to the Tmote using serial communication. The Tmote routes the information to the base station using multihop communication. Both the Cyclops and the Tmote run the TinyOS operating system.

We collected data using the SCOPES system for a period of 24 hours and compared them to ground-truth data. To record ground truth data, we installed three Philips SPC$900 \mathrm{NC}$ web cameras to record the movement of people. The camera captured approximately three images every two seconds. This frequency is high enough to record transitions accurate to the second. We collected data from the ground truth system for the entire 24 hours that the SCOPES system was operated and then for an additional 24 hours. 
Overall, SCOPES detected $80 \%$ of all recorded transitions. However, the system has false positives of $25 \%$. False positives refer to the ratio of number of transitions detected by SCOPES when the ground truth shows none to the total number of transitions detected by SCOPES. False positives result, mainly, due to background noise, and difficulties with hardware calibration.

The data from the resulting network were used to test and evaluate the performance of occupancy estimation and people dynamics (Tasks 4 and 5).

Further details of the SCOPES system are available in Erickson et al., 2009 and a report by A. Cerpa to CEC (in preparation). 

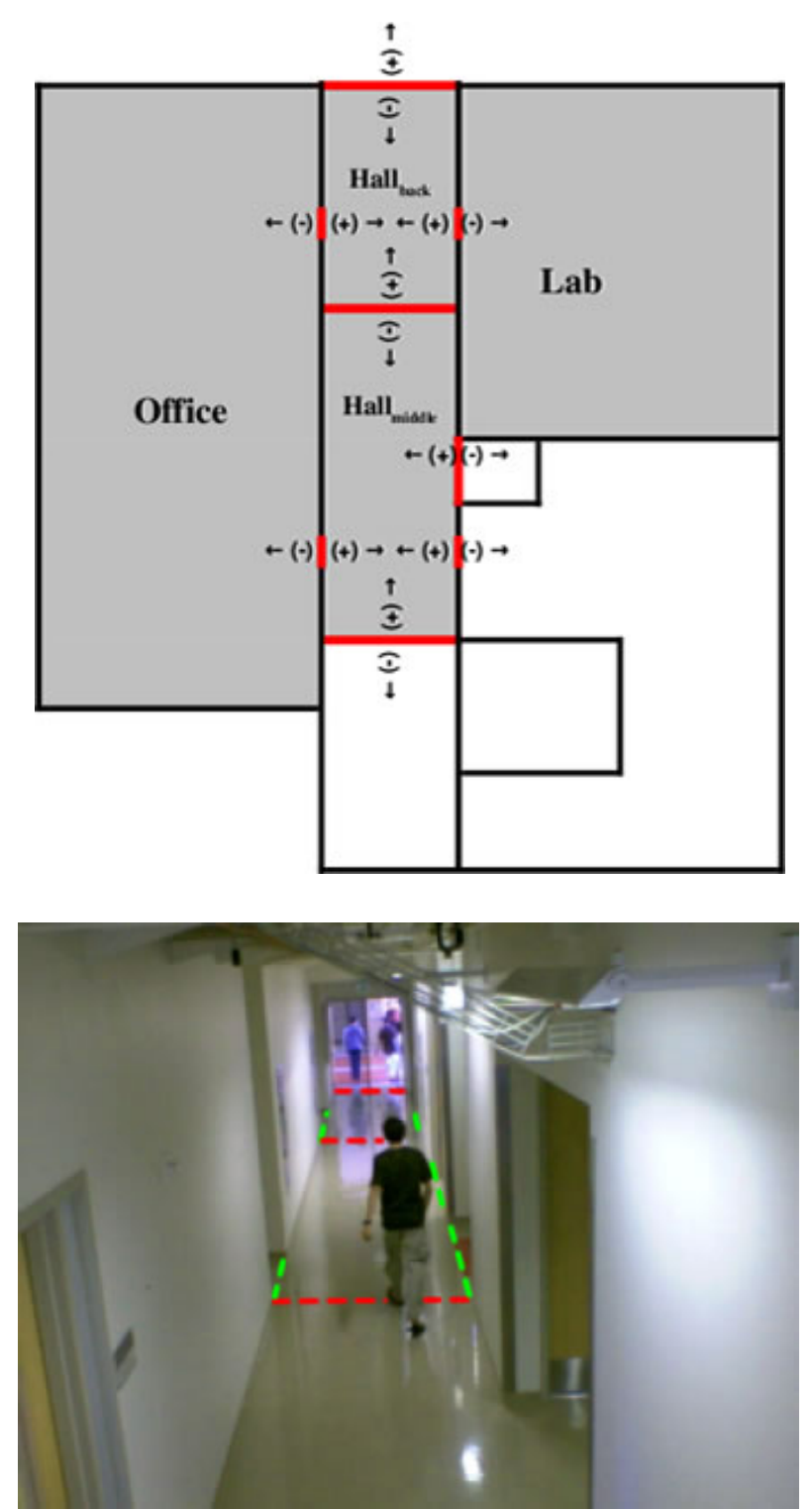

Figure T3-1: In the photo, the red and green lines indicate the locations of the transition boundaries. In the graphic, the arrows show the sign associated with each transition direction. In the graphic, the gray areas indicate the area occupancies that can be derived from the transition data. 


\section{Task 4: Develop Energy Controls for the COB (UTRC Lead Author)}

Traditionally, lighting, temperature, and ventilation (outside air - OA) controls are based on schedules, and set according to expected, or assumed, room usage. However, control is often based on maximum capacity, which frequently overestimates actual use. In this task, we conduct an order-of-magnitude estimation of energy-savings potential if we know occupancy using the COB as a study example.

We employed a model-based order-of-magnitude estimation: we first generated a suite of typical building occupancy patterns; set building controls, such as setpoints, based on the occupancy; and estimated energy savings using an eQuest model of the COB.

Lastly, we tested the feasibility of estimating real-time occupancy in the Science \& Engineering building at UC Merced campus, since the building was instrumented with a network of wireless cameras that is able to collect people traffic data.

\section{Energy Model}

We used an existing eQuest model of the COB because the EnergyPlus model (Task 2) was not yet available. The model was developed by Taylor engineering for the design and construction of the COB. The model has less temporal resolution than the EnergyPlus model, 1 hour vs. 15 minutes, but we felt it was suitable for the order-ofmagnitude calculation. We planned to use the EnergyPlus model in future phases of the project.

\section{Occupancy Schedules}

EQuest uses the occupancy to specify outside air quantities. In the for-building-design model, building occupancy for classrooms and offices uses one class or office schedule, respectively, for all seasons.

We updated the schedules that better describe actual classroom and office use: each classroom has a unique schedule, which is based on the UC Merced schedule of classes for each week, including summer and holiday periods, and each office zone (group of offices) has its own occupancy schedule, which we generate based on an occupancy movement model (Task 5). Minimum flow in spaces where occupancy is not known is $40 \%$ in classrooms, auditoriums, and conference rooms and $30 \%$ in offices. Figures T4-1 and T4-2 show classroom and office occupancy schedules.

\section{Control Strategies}

We simulated three ventilation control strategies: - base, current, and new. In the base case the OA quantity is based on maximum design occupancy, and is fixed, irrespective 
of actual occupancy in individual zones/rooms. This control strategy is found in many buildings. The second control strategy, which we call current, emulates the actual strategy used in the $\mathrm{COB}$ presently. It is based on information from $\mathrm{CO}_{2}$ sensors in classrooms, conference rooms, and auditoriums (spaces with high variability in occupancy), which account for two-thirds of the building space. If $\mathrm{CO}_{2}$ readings in any zone are beyond the acceptable limit, the sensor triggers a request for more OA from the air handling unit (AHU). The remaining zones (primarily office spaces) in the building are not instrumented with sensors that can be used to discern actual occupancy levels, and the control strategy therefore defaults to assuming maximum occupancy level in these zones. The simulation of the "current" strategy was set up to reflect dynamic knowledge of the occupancy levels only in the spaces instrumented with $\mathrm{CO}_{2}$ sensors. Essentially this assumes $\mathrm{CO}_{2}$ level measurements are reasonably accurate surrogates for actual occupancy level in indoor spaces (Stanke, 2006). Lastly, the third control strategy, which we call new, adjusts OA in all zones (except restrooms, hallways, and service spaces) according to actual (predicted) occupancy in the respective zones. This scenario is equivalent to the current strategy plus knowing occupancy in the unmonitored zones. The HVAC control is therefore based on knowledge of actual occupancy throughout the building, e.g. from $\mathrm{CO}_{2}$ sensors in all offices, classrooms, conference rooms and auditoriums (except restrooms, hallways, and service spaces). Control strategies account for building code requirements (both Title 24 and ASHRAE 62.1 2007).

\section{Results}

Any method to reduce fresh air ventilation from the base case, while meeting air quality standards, will result in significant energy savings. Tables T4-1 and T4-2 show aggregate energy savings for each control strategy. As compared to the base control strategy, the occupancy-based control strategies results in a total building energy consumption reduction of approximately 3-5\% depending on the applicable ventilation code requirements (AHRAE 62.12007 and Title 24). Energy savings from HVAC operation ranges between $10-14 \%$ from the base case. The results are similar to estimates reported in the published literature, for example Brandemuehl MJ and Braun, 1999. We see most of the energy savings from reducing the heating of unoccupied spaces. As expected, knowing the occupancy levels in classrooms, conference rooms and auditorium spaces results in the majority of the savings (as reflected in the figures quoted for the "current control strategy" which utilizes $\mathrm{CO}_{2}$ sensors in the $\mathrm{COB}$ for zonal occupancy level estimation in a portion of the building). This is because the number of occupants in such zones is much more than in the office area and there is significant amount of variability in this area's occupancy (Figure T4-1, T4-2).

There are incremental savings that result from knowledge of occupancy levels in COB zones that are currently not instrumented with relevant sensors (e.g. $\mathrm{CO}_{2}$ or other occupancy level sensors). The tables show that incremental savings of $4 \%$ (beyond the "current control strategy" case) are possible if dynamic knowledge of actual occupancy levels in all the zones in the building is available. This could be accomplished simply by 
instrumenting all the zones in the building with $\mathrm{CO}_{2}$ sensors or using more cost effective sensors with comparable accuracy (e.g., cameras). If full instrumentation is cost prohibitive, advanced estimation algorithms can be used (such as evaluated in the Science and Engineering Building) that can exploit prior knowledge of occupancy patterns in the building (via models or schedule knowledge) and infer occupancy levels in zones where no sensors or poor quality sensors are available. We explore these options in Task 5 and in future phases of the project. 
Table T4-1: Saving calculations based on ASHRAE 62.1 2007 ventilation code

\begin{tabular}{|c|c|c|c|c|c|c|c|c|c|}
\hline Values in MMBtu & Heating & Cooling & $\begin{array}{c}\text { Heat } \\
\text { Reject }\end{array}$ & Pumps & Fan & HVAC & $\begin{array}{c}\% \\
\text { Savings } \\
\text { HVAC }\end{array}$ & Total & $\begin{array}{c}\% \\
\text { Savings } \\
\text { Total }\end{array}$ \\
\hline Base Control Strategy & 335 & 252 & 5 & 150 & 128 & 870 & - & 2578 & - \\
\hline Current Control Strategy & 327 & 214 & 4 & 141 & 101 & 786 & $10 \%$ & 2494 & $3.3 \%$ \\
\hline New Control Strategy & 293 & 212 & 4 & 140 & 101 & 750 & $14 \%$ & 2457 & $4.7 \%$ \\
\hline
\end{tabular}

Table T4-2: Saving calculations based on Title 24 ventilation code

\begin{tabular}{|c|c|c|c|c|c|c|c|c|c|}
\hline Values in MMBtu & Heating & Cooling & $\begin{array}{c}\text { Heat } \\
\text { Reject }\end{array}$ & Pumps & Fan & HVAC & $\begin{array}{c}\% \text { Savings } \\
\text { HVAC }\end{array}$ & Total & $\begin{array}{c}\text { \% Savings } \\
\text { Total }\end{array}$ \\
\hline Base Control Strategy & 308 & 222 & 4 & 133 & 119 & 785 & - & 2493 & - \\
\hline Current Control Strategy & 259 & 207 & 3 & 130 & 97 & 696 & $11 \%$ & 2404 & $3.6 \%$ \\
\hline New Control Strategy & 243 & 206 & 4 & 129 & 94 & 676 & $14 \%$ & 2383 & $4.4 \%$ \\
\hline
\end{tabular}




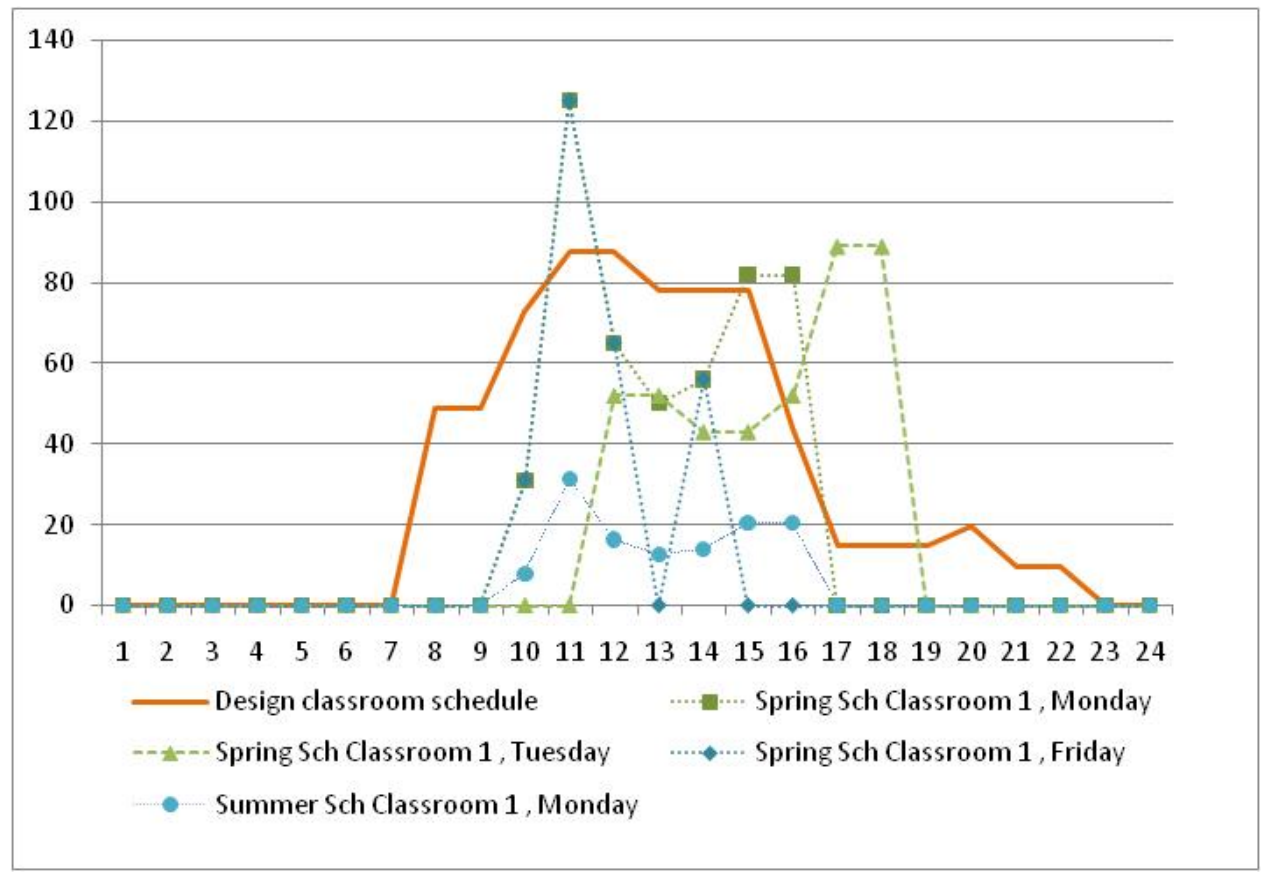

Figure T4-1: Classroom schedules - design stage assumption versus schedules based on actual UC Merced class schedules. The x-axis is hours and the $y$-axis are number of people. 


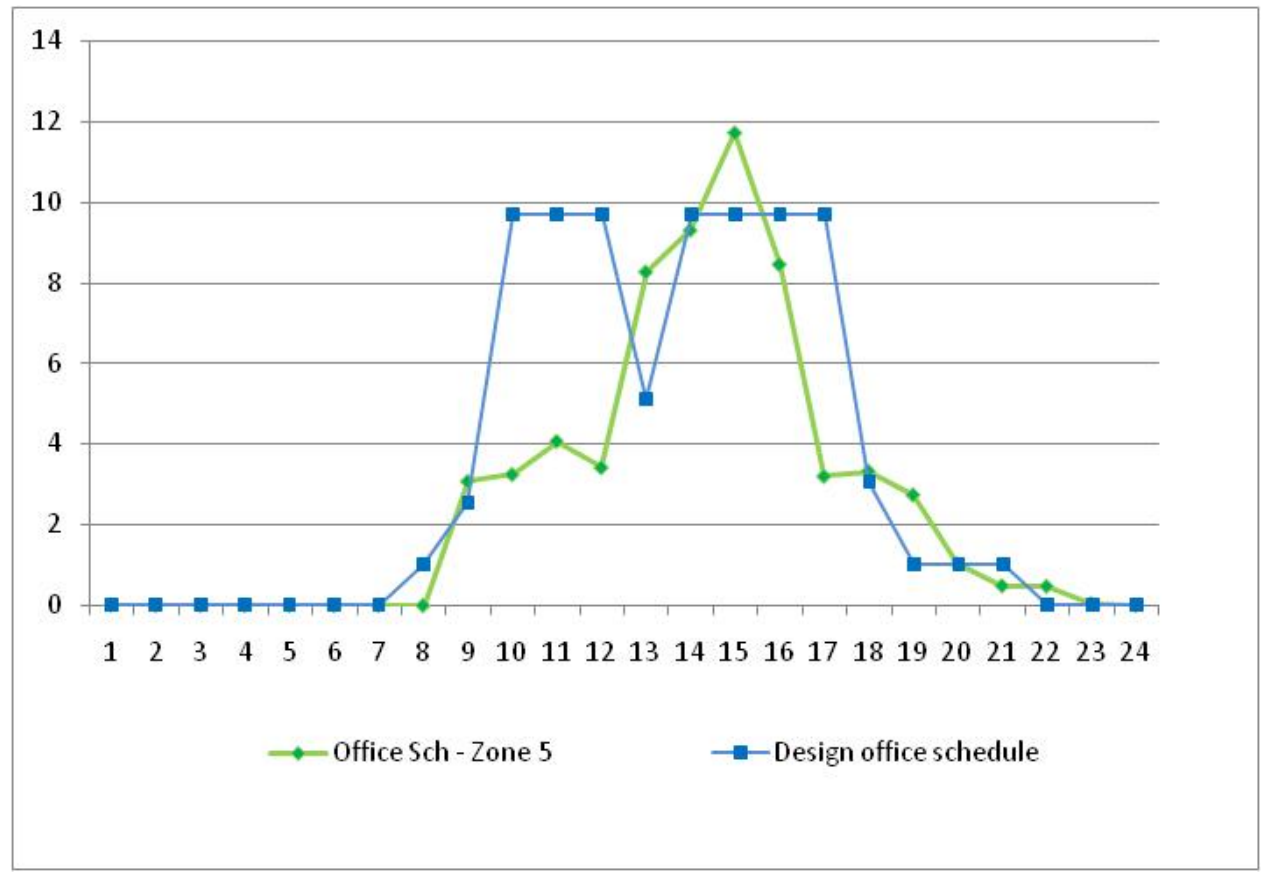

Figure T4-2: Office schedules - design stage assumption versus schedule derived from occupancy movement model (Task 5). The x-axis is hours and the y-axis are number of people. 


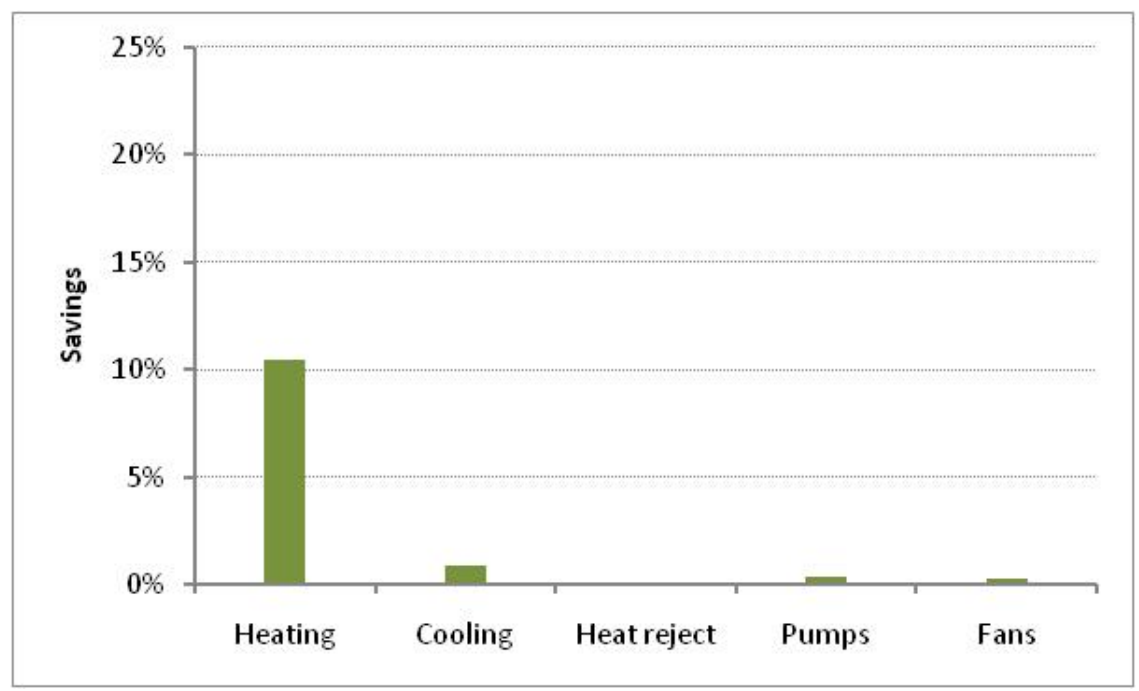

Figure T4-3: Percent energy savings of new (occupancy-based) control strategy compared to current $\mathrm{COB}$ control strategy. 


\section{Task 5: Develop People Dynamics Model for Building Occupants (UTRC Lead Author)}

The goal of this task is to study the feasibility of estimating occupancy and people dynamics. This task complements Task 4 by providing the level of occupancy knowledge obtainable in a typical building system. We use existing technology at UTRC (occupancy modeling) and UC Merced (camera-based occupancy estimation and sensor-network hardware in the S\&E Building) to calculate rough-order-of-magnitude calculations.

\section{Approach}

The overall framework is shown in Figure T5-1. For this task, we focused on applying an occupant-movement model and the SUN (Sensor Utility Network) estimator to the UC Merced buildings.

The occupant movement model is a high resolution simulation model which simulates each individual's movement at fine spatial and temporal scales. The model is able to provide quantitative output such as occupancy and congestion levels at any location and at any desired time period (Lin 2007). The simulation model generated occupancy levels of the office area in the Classroom and Office Building. Model parameters were computed from the wireless camera sensor network data collected in the S\&E Building (Task 3). The resulting occupancy predictions were used for the energy savings analysis (Task 4).

For occupancy-based energy management and control, real time occupancy estimation is required at a somewhat coarse spatial scale (e.g., HVAC zone level). UTRC developed the SUN estimation framework (Meyn et al. 2009) for this purpose. Based on inputs from a variety of sensor measurements and sensor characteristics, along with historical data regarding building utilization, the SUN estimator produces occupancy estimates through a solution of a receding-horizon convex optimization problem.

Details of the occupant estimation model are published in (Meyn et al. 2009). The general approach generates occupancy estimates based on a combination of sensor data, information regarding prior knowledge of building utilization, and the network structure of the building. Sensors may include $\mathrm{CO}_{2}$ sensors, passive infrared (PIR), video, sound, and badge counters. The utilization of the building, and the zones, is estimated based on historical data for the building or a building of similar characteristics, forecast preferences based on room schedules, typical walking speeds in hallways, and hard constraints on occupancy in each room. Finally, the network structure is specified by decomposing the total building into zones, typically based on individual rooms or groups of rooms. The choice of zones will depend on the primary goal (e.g., ventilation control vs. evacuation), and on the number, location, type and accuracy of the sensors. 
Estimation is then based on solution of an optimization problem with hard and soft constraints, and taking into account the inherent bias and variance of the various sources of information. The output of the model is the occupancy and flow level estimation.

\section{Results}

We show an implementation of the estimator for a portion of the $2^{\text {nd }}$ floor S\&E building (Figure T5-2). UC Merced collected 11 hours of sensor data. The sensors estimate the number and time when people pass under a low-resolution camera (see Task 3 ). For the 11-hour sequence of data, the sensors were on average $80 \%$ accurate, as compared to corresponding ground truth data from a high-resolution video camera. The occupancy estimator processes the sensor data to estimate occupancy for the 11 hours at 10-minute intervals. For the first run of the model, initial occupancy and flow level are set to zero. For each of the next runs, initial conditions for the preceding optimization is derived from the estimate from the previous optimization.

Figure T5-2 shows the occupancy variation for the area. The red curve denotes the ground truth occupancy, black curve is the estimate, cyan curve is an estimate derived directly from sensor data by simple flow balance, and the green curve is also an estimate based on flow balance but with the additional constraints on non-negativity of occupancy. We show that the SUN estimator is able to provide occupancy estimates with significantly improved accuracy compared to estimates derived from simply peopleflow balancing from the sensor data. The zone-level error from the SUN estimator is $35 \%$.

In addition to the sensor data provided by the UC Merced team, simulated sensor data was generated for a larger area of the S\&E building using a calibrated occupant movement model (Lin 2007). Figure T5-3 shows improved accuracy (by almost $80 \%$ ) compared with the estimates derived directly from sensor data.

Additional tests were conducted to evaluate the effect of passive infrared (PIR) sensors on the performance of the SUN estimator. Specifically, in addition to the existing sensor data provided by cameras, we evaluated the benefit of using additional PIR sensors in the estimation. The PIR sensor data was generated from ground truth data with no noise in the PIR output. The overall estimation improves by approximately $35 \%$ with the addition of PIR sensors. 


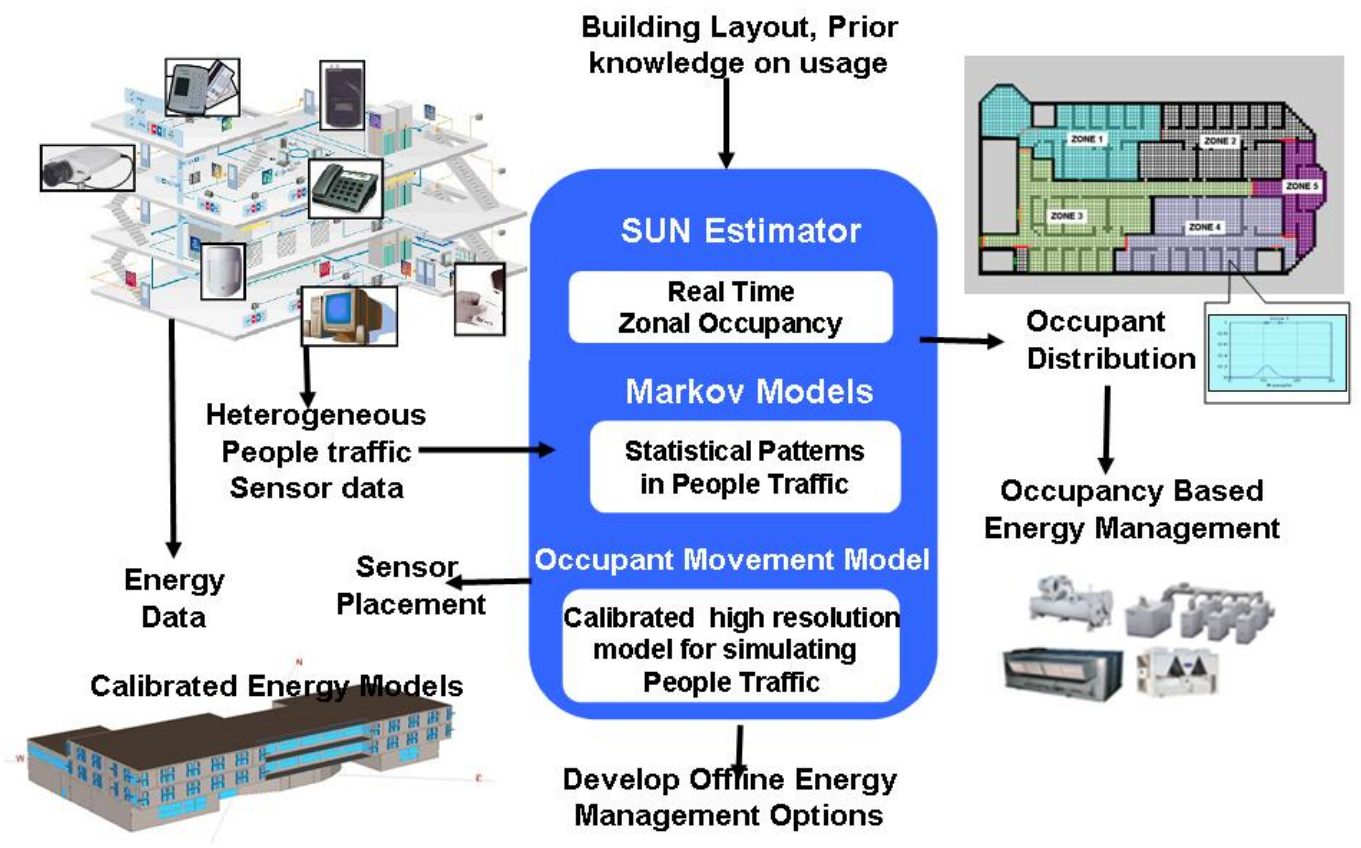

Figure T5-1: Overview of Occupancy Modeling and its applications 

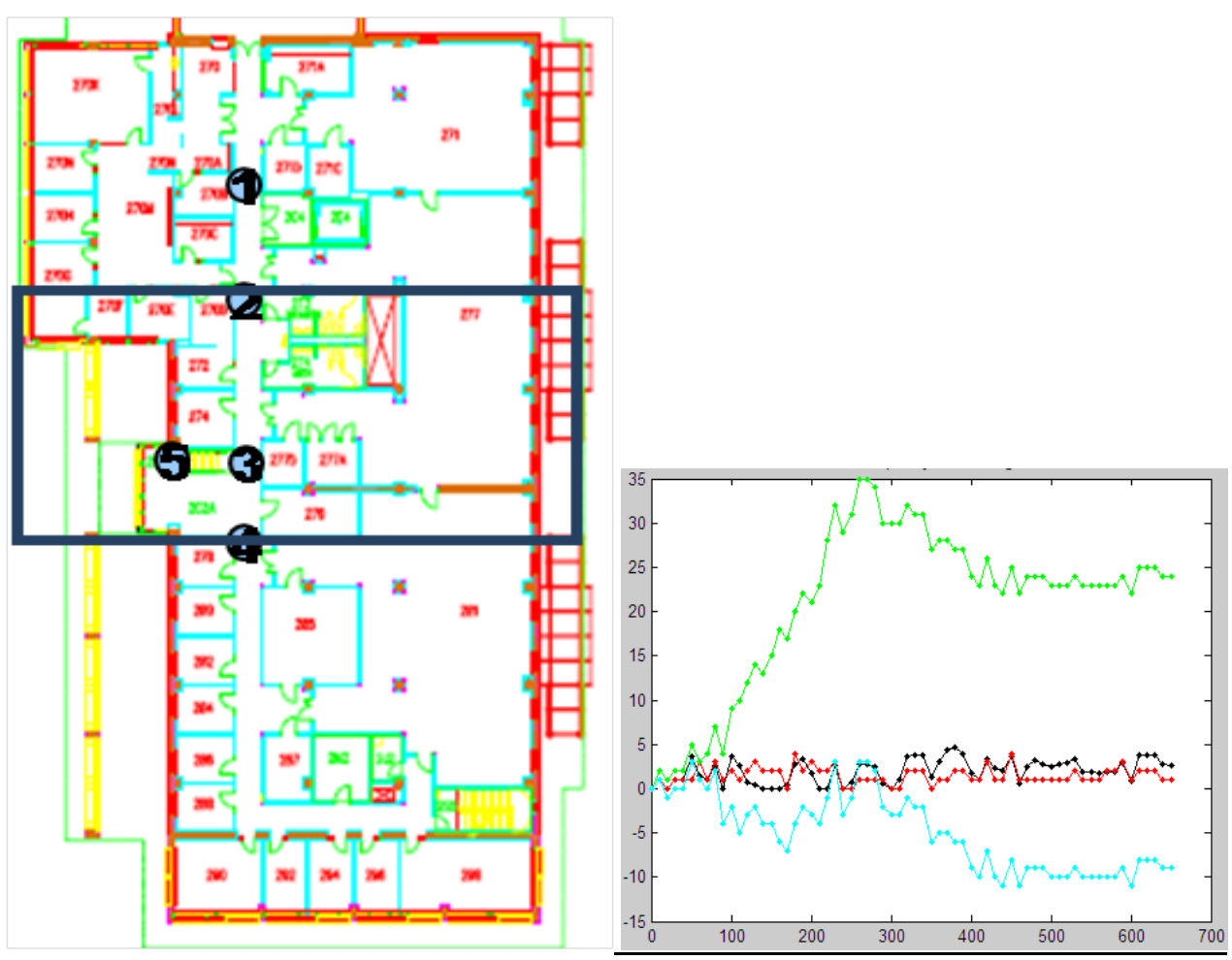

Figure T5-2: Area (defined in black box) in the short bar of $2^{\text {nd }}$ floor S\&E building used for estimation. Ground truth (in red) vs. estimated occupancy using different approaches. 


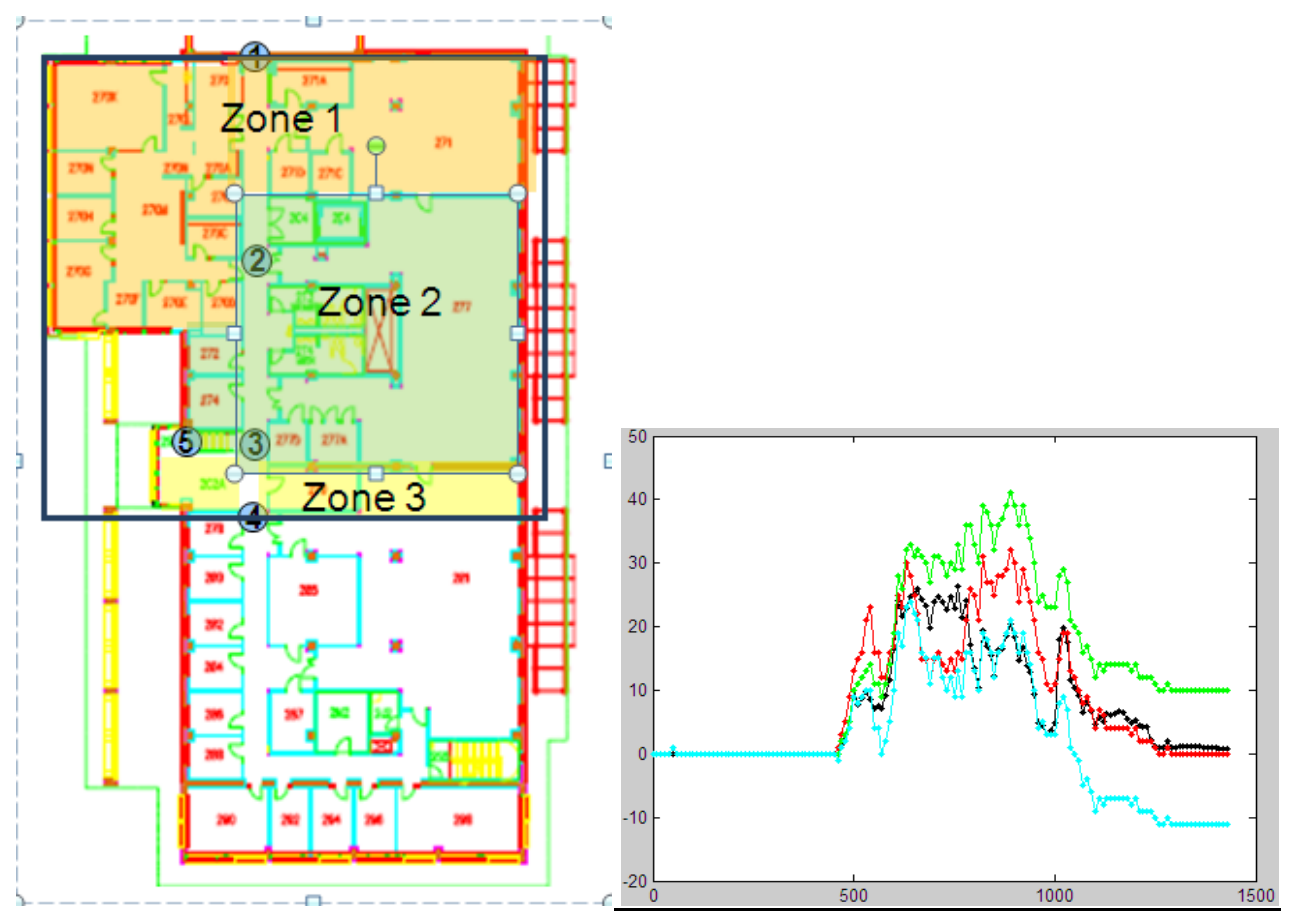

Figure T5-3: Estimated occupancy for the area contained within the black box. The ground truth (in red) vs. estimated occupancy using different approaches every 10 minutes for 24 hours. 


\section{Discussion and Concluding Remark}

We report on the findings of the first year of a planned multi-year project. In the first year, our goal was to benchmark the likely energy benefits of knowing the number and location of occupants in a typical commercial building, and the feasibility of estimating occupancy. To do so, we conducted five tasks:

1. Describe energy usage in the $\mathrm{COB}$;

2. Develop an energy model of the COB;

3. Deploy a sensor network in the S\&E;

4. Develop energy controls for the COB; and

5. Develop a people dynamics model for occupants in the S\&E.

As proof of concept, we studied a model of the COB and found that knowing the number of building occupants in each zone can result in HVAC energy consumption reduction of nearly $15 \%$, when compared to a HVAC control strategy that assumes full building occupancy at all times in all the zones. It was not possible to distinguish the savings that result from real time knowledge of the zonal occupancy levels from those due to knowledge of occupant location, and is a subject of future research. A 16-node wireless camera sensor network was deployed in a multi-function building to determine the occupancy resolution one can conceive of obtaining in a typical building. Finally, we developed an EnergyPlus model of the $\mathrm{COB}$ to support the above tasks and tasks on a related project (Real-Time Visualization of Energy Performance in Buildings (Piette et al., 2010)).

Subsequent tasks and funding for Phases 2 and 3 will be sought after further study of the results from Phase 1. There are several challenges both from implementation and theoretical perspectives that need to be addressed. For instance, the heuristic approach to learn traffic patterns from a building area and using it for another building area of similar usage needs to be experimentally validated. In addition, the real time feasibility of occupancy based adaptive energy management and control remains to be evaluated in a test-bed. On the theoretical side, one needs to address issues such as: adaptive techniques for learning building usage and associated utility functions, sensitivity of utility functions for spaces and buildings of similar type, and optimal sensor placement (numbers, types and locations) for occupancy estimation. Finally, decentralized algorithms are required to make the occupancy estimation scalable and reliable in large buildings.

\section{Acknowledgements}

This work was supported in part by the Department of Energy and the California Energy Commission, and performed under U.S. Department of Energy Contract No. DE-AC02$05 \mathrm{CH} 11231$ and the California Institute for Energy and the Environment under contract 
No. MUC-09-03. We are grateful to Dr. Andrzej Banaszuk (UTRC) for valuable technical guidance on modeling and estimation of occupancy dynamics.

\section{References}

Apte M. G., 2006; A review of demand Control ventilation. Lawrence Berkeley National Laboratory, LBNL-60170, May 2006

Brandemuehl MJ and Braun JE. The impact of demand-controlled and economizer ventilation strategies on energy use in buildings. ASHRAE Transactions, 105(2), 1999.

Bierlaire G. A., M and M. Webers. Behavioral dynamics for pedestrians, 2003; In 10th International Conference on Travel Behavior Research, Aug. 2003.

Dietz, B. and J. Wilkerson; UC Merced Classroom and Office Building Data Comparison

Erickson V, Lin Y, Kamthe A, Brahme R, Cerpa A, Sohn MD, Narayanan S. 2009. "Energy Efficient Building Environment Control Strategies Using Real-time Occupancy Measurements," in proceedings of the ACM BuildSys conference, Berkeley, California, USA, November 4 - 6, 2009.

A.Kamthe, L.Jiang, M.Dudys, and A.Cerpa. SCOPES: Smart Cameras Object Position Estimation System. In In the Proceedings of the 6th European Conference on Wireless Sensor Networks(EWSN 2009), pages 279-295, Cork, Ireland, Feb. 2009. SpringerVerlag.

Lin, Yiqing, Agent-Based Model for People Traffic under Normal Condition, UTRC technical report, 2007.

Meyn S. P., Y. Lin, S. M. Oggianu, A. Surana, and I. Fedchenia, 2008; System and method for occupancy estimation and monitoring. USPTO patent application under review, 2008.

Meyn S. P., A. Surana, Y. Lin, S. M. Oggianu, S. Narayanan, T. Frewen, 2009; A sensor-utility-network method for estimation of occupancy distribution in buildings. In Proceedings of IEEE Conference on Decision and Control, Shanghai, China, 2009.

Payne FW. Energy management control system handbook. Fairmont Press, 1984.

Piette, Apte, et al 2010. Real-Time Visualization of Energy Performance in Buildings. LBNL Report (draft). 
Schadschneider A., 2002; Pedestrian and Evacuation Dynamics, chapter Cellular automaton approach to pedestrian dynamics theory, pages 75-86. Springer, 2002.

Stanke, D. Standard 62.1-2004 System Operation: Dynamic Reset Options. ASHRAE Journal, December 2006.

Taylor engineering, 2002; University of California, Merced Academic Building, Detailed Energy Analysis Report, December 30, 2002. 


\section{Appendix: Publications Resulting from Research}

1. Erickson V, Lin Y, Kamthe A, Brahme R, Cerpa A, Sohn MD, Narayanan S. 2009. "Energy Efficient Building Environment Control Strategies Using Real-time Occupancy Measurements," in proceedings of the ACM BuildSys conference, Berkeley, California, USA, November 4-6.

2. Narayanan S, Apte MG, Haves P, Piette MA, and Elliott J. 2010. "Systems Approach to Energy Efficiency Building Operation: Case Studies and Lessons Learned in a University Campus," in proceedings of ACEEE 2010 Summer Study for Building Energy Efficiency, Pacific Grove, California, USA, August 15-20. 


\title{
Energy Efficient Building Environment Control Strategies Using Real-time Occupancy Measurements
}

\author{
Varick Erickson ${ }^{1}$, Yiqing Lin $^{2}$, Ankur Kamthe ${ }^{1}$,Rohini Brahme ${ }^{2}$, \\ Alberto Cerpa ${ }^{1}$, Michael D. Sohn ${ }^{3}$ and Satish Narayanan ${ }^{2}$ \\ ${ }_{1}^{1}$ University of California - Merced \{verickson, akamthe,acerpa@ucmerced.edu\} \\ ${ }^{2}$ United Technologies Research Center \{LinY,BrahmeR,NarayaS@utrc.utc.com\} \\ ${ }^{3}$ Lawrence Berkeley National Laboratory \{MDSohn@lbl.gov\}
}

\begin{abstract}
Current climate control systems often rely on building regulation maximum occupancy numbers for maintaining proper temperatures. However, in many situations, there are rooms that are used infrequently, and may be heated or cooled needlessly. Having knowledge regarding occupancy and being able to accurately predict usage patterns may allow significant energy-savings by intelligent control of the L-HVAC systems. In this paper, we report on the deployment of a wireless camera sensor network for collecting data regarding occupancy in a large multi-function building. The system estimates occupancy with an accuracy of $80 \%$. Using data collected from this system, we construct multivariate Gaussian and agent based models for predicting user mobility patterns in buildings. Using these models, we can predict room usage thereby enabling us to control the HVAC systems in an adaptive manner. Our simulations indicate a $14 \%$ reduction in HVAC energy usage by having an optimal control strategy based on occupancy estimates and usage patterns.
\end{abstract}

\section{Introduction}

Heating ventilating and air conditioning (HVAC) systems account for 50\% of the total energy budget in buildings [5]. Prior research has shown that energy savings are achievable by regulating fresh air ventilation based on the total number of occupants in a building. This procedure is referred to as demand-controlled ventilation, and studies suggest 10 to $15 \%$ of HVAC energy can be reduced in buildings that set ventilation rates based on maximum occupancy [2].

In general, the approach used is to assume that all rooms are occupied during working hours and not being used during the night. However, it is obvious that this does not maximize energy savings. Rooms are often left empty during part of the day or perhaps are only used semi regularly, e.g. con-

Permission to make digital or hard copies of all or part of this work for personal or classroom use is granted without fee provided that copies are not made or distributed for profit or commercial advantage and that copies bear this notice and the full citation on the first page. To copy otherwise, to republish, to post on servers or to redistribute to lists, requires prior specific permission and/or a fee. ference rooms. It would be more efficient to only condition rooms during the times that are actually occupied. Using an L-HVAC system (lighting, heating, venting, and air conditioning system), various environmental aspects of room can be controlled for energy savings. Thus, knowledge of occupancy is crucial in order to maximize efficiency of a system.

If a room is known to be occupied, then specific strategies can be used to condition a room optimally. The lack of reliable data (both real time and model) for the movement of people inside buildings may make many of these aspects difficult to control efficiently. Although many modern buildings include motion detectors and temperature and $\mathrm{CO}_{2}$ sensors for light and air flow management, these sensors present some limitations. Motion detectors provide an efficient way to detect occupancy, but they provide no information about the quantity of people using the space since their output is a binary process. While temperature and $\mathrm{CO}_{2}$ sensors may provide better indirect measures of actual occupancy, the physical phenomena being measured responds to different time scales and these sensors may be more suitable for understanding general trends at large time scales. A faster new distributed sensor network may be needed to respond quickly to ever changing occupancy. Therefore, conditioning of the room must begin prior to when the room is actually utilized. Thus, having the capability to predict user movement or room usage patterns prove to be helpful for demand-control ventilation; they may also provide enough information for even substantial local-scale controls. Occupancy prediction can be achieved by modeling long traces of occupancy data captured by a sensing system [6], helping modify L-HVAC control strategies as building usage and occupancy patterns change with time.

In this paper, we describe the experiments conducted with SCOPES [4], a wireless camera sensor network for gathering traces of human mobility patterns in buildings. With this data and knowledge of the building floorplan, we created two prediction models for describing occupancy and movement behavior. The first model comprises of fitting a Multivariate Gaussian distribution to the sensed data and using it to predict mobility patterns for the environment in which the data was collected. The second model is an Agent Based Model (ABM) that can be used for simulating mobility patterns for developing HVAC control strategies for buildings that lack an occupancy sensing infrastructure. We integrate the sim- 
ulation information to compute energy saving for a building where we can adjust L-HVAC controls based on occupancy estimates from a sensor network.

The paper is organized as follows: Section 2 describes the wireless camera sensor network used for occupancy estimation. Section 3 describes how occupancy data is used to develop models for user mobility prediction and energy saving estimation and Section 4 compares the performance of these models. Section 5 discusses the L-HVAC control strategies and energy savings. Section 6 summarizes our paper and discusses future work.

\section{Occupancy Sensing}

\subsection{Hardware and Software Infrastructure}

We deployed the SCOPES system [4] comprising of 16 sensor nodes on the ceiling of the corridors in the University of California - Merced Science and Engineering building. The nodes were deployed in groups of three at transition points of the floorplan. Each sensor node is comprised of a $\mathrm{Cy}$ clops camera interfaced with a Moteiv Tmote Sky module via an intermediate adapter board. The Cyclops is a very low power camera with an on-board $4 \mathrm{MHz}$ ATmega128L micro-controller (MCU) and 512KB of external SRAM. Due to the limitation of the total addressable memory, the external SRAM is divided into eight, 64KB memory banks. The Cyclops captures $1064 \times 64$ pixel grayscale images per bank (i.e., 80 total). In each group, multiple nodes sense the same area with coordination such that there is minimal overlap between the image capture periods of the cameras. Whenever a person crosses any one of these transition points, the cameras capture and process the image data and, thus indicating whether the transition was recorded or not.

Due to the severe limitations on the available computational power, we execute lightweight processing algorithms on the Cyclops. The object detection algorithm running on the Cyclops determines the presence of an object in the image foreground, if any, and updates the background. This is done using a modified background subtraction algorithm that labels the pixels in the captured images as object, shadow or background depending upon a preset threshold. Next, we use a connected components algorithm to merge all pixels labelled as object into a blob. For each blob, information regarding the centroid ( $\mathrm{x}$ and $\mathrm{y}$ coordinates) and the number of pixels is recorded. We track the movement of the blob across images based on displacement with respect to the previous image. After processing all the images in the current bank, an array of data structures containing information on a maximum of four objects per bank is transferred to the Tmote using serial communication. The Tmote routes the information to the base station using multihop communication. Both the Cyclops and the Tmote run the TinyOS operating system.

\subsection{System Evaluation and Data Verification}

We collected data using the SCOPES system for a period of 24 hours. This was done to evaluate the performance of the system with respect to the ground truth. Overall, the SCOPES system would detect $80 \%$ of all recorded transitions over a period of 24 hours. The system has a false positive rate of $40 \%$ which was higher than the one encountered in the 2-hour experiments conducted in [4].
For collecting the ground truth to compare the performance of SCOPES, we installed three Philips SPC-900NC web cameras to record the movement of people. The camera captured approximately three images every two seconds. This frequency is high enough to record transitions across areas of interest to the accuracy of seconds. The ground truth data was processed using Perlmagick which annotates images with people in them. We manually corrected the Perlmagick output for the false positives and false negatives in the processed ground truth. We collected data from the ground truth system for the entire 24 hours that the SCOPES system was operated and then for a further 24 hours. Our goal was to use the SCOPES dataset to characterize the sensor error and the ground truth data for creating models of user mobility patterns.

From the ground truth data, we defined transition points in the floorplan (refer Figure 1). The time, direction, and number of people transitioning were recorded for each transition. The sign of the transition is used to indicate direction of travel. The transitions points are strategically placed at entrances/exits to capture the occupancy changes of each area.

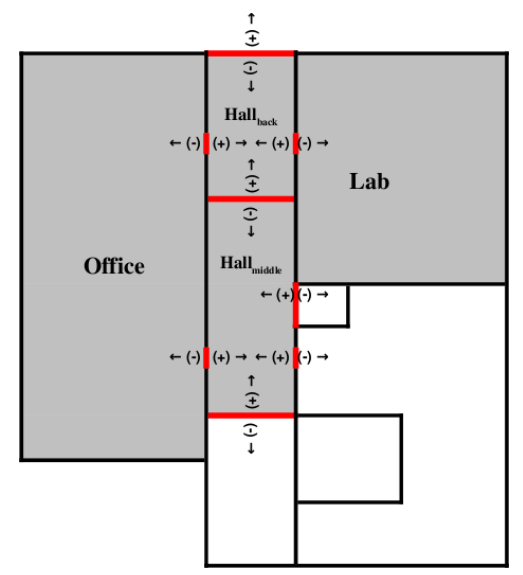

(a) Occupancy Areas

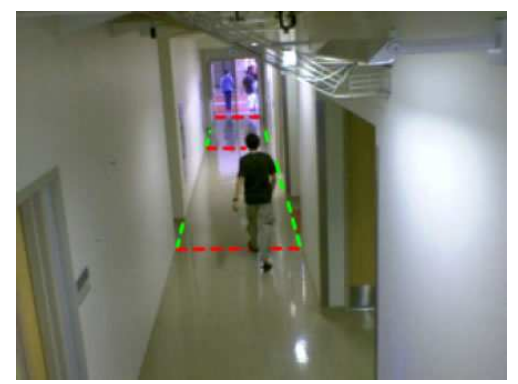

(b) Sample Camera Image

Figure 1. The red and green lines indicate the locations of the transition boundaries. The arrows show the sign associated with each transition direction. The gray areas indicate the area occupancies that can be derived from the transition data.

\section{Occupancy Models}

Understanding the dynamics of occupancy patterns is central to the approach of occupancy-based building energy 
management. Multi-scale spatiotemporal dynamics of occupancy with high variability makes this a challenging task. In this paper, we developed two dynamic models of occupancy distributions, the Multivariate Gaussian Model and the Agent-Based simulation Model (ABM). These models enable simulations at an individual level and are useful for offline studies, such as: 1) learning occupancy patterns from sensor data and generating variations of those patterns which can be used for evaluating different energy management options, 2) optimal sensor placement for accurate estimation of occupant traffic, and 3) learning statistical patterns in occupant traffic in one building and applying it to other buildings of similar types to simulate occupancy dynamics.

\subsection{Multivariate Gaussian Model}

In this section we discuss a simple occupancy model that utilizes multiple gaussian fits over the data. In particular, we focus on constructing a model that can simulate the occupancy for two specific areas that are represented by the ground truth data. Given its simplicity, this model serves as a coarse baseline model for other occupancy simulation and prediction approaches.

\subsubsection{Training}

On an intuitive level, over the course of a day we expect occupancy to increase in the morning when people arrive for work, decrease when people go to lunch, increase when people return from lunch, and then eventually drop to zero when people leave for the day. These are the general increases and decreases of occupancy we can expect based on our real world experiences. The strategy behind this approach is to model each of the increases and decreases separately.

However, as the occupancy data suggests in Figure 2, there may be other regular phenomenon affecting occupancy other than those based on intuition. In order to ensure that the majority of occupancy dynamics is captured, the data is partitioned into hourly blocks.

Let $O_{h}$ denote all occupancies that occur per second during hour $h$ where $1 \leq h \leq 24$,

$$
O_{h}=\left\{\begin{array}{cccc}
H B_{1} & H M_{1} & L_{1} & O f_{1} \\
\vdots & \vdots & \vdots & \vdots \\
H B_{n} & H M_{n} & L_{n} & O f_{n}
\end{array}\right\}
$$

$H B, H M, L$, and $O f$ refers to the Hallway ${ }_{b a c k}$, Hallway ${ }_{\text {middle }}$, Lab, and Office areas occupancies respectively (refer Figure 1). $n$ represents the number of observations that occur during hour $h$ within the training set.

Using the data $O_{h}$, we derive a vector of means $m_{h}$ and covariance matrix $\sigma_{h}$ that fit the data to a multivariate normal distribution $X_{h}$ :

$$
X_{h}=N\left(m_{h}, \sigma_{h}\right)
$$

For hours 8:00:00 to 22:00:00, we compute $X_{h}$. These are the hours that have the most occupancy dynamics. For hours 23:00:00 to 7:00:00, we assume that the occupancy for all areas is zero. This assumption is based on our observation of the 48 hours ground truth and intuition. For simulation we can randomly draw occupancies from $X_{h}$. We are also able to use the probability density function (pdf) of $X_{h}$ to estimate the probability of an occupancy occurring within a given hour $h$.

\subsubsection{Simulation Generation}

However, randomly drawing occupancies from $X_{h}$ does not represent certain constraints. For example, suppose we randomly sample from the distribution and obtain $\left\{\begin{array}{llll}0 & 0 & 0 & 0\end{array}\right\}$ for time $t$ (all areas are empty) and $\left\{\begin{array}{llll}0 & 0 & 0 & 1\end{array}\right\}$ for $t+1$ (one person in the office). This situation is impossible since a person must first pass through the hallway to reach the office. Thus, if we sample randomly from the fit distributions without restrictions, the simulation will produce many occupancy transitions that are impossible.

In order to solve this problem, rather than sample from the entire distribution, we instead randomly sample from a subset of the distribution. We only sample occupancies that are actually possible given the current occupancy state. The following is algorithm we follow to generate simulations:

- CurrentOcc $=$ Specify starting occupancies

- StartHr = Specify starting hour

- $p d f(X, o c c)=$ Probability of an occupancy occ given a multivariate normal distribution $X$.

- For each simulated timestep $t$

- CurrentHr $=$ Current hour based on $t$ and StartHr

- If $8 \leq$ Current $H r \leq 22$

- ValidOcc = All valid occupancies given CurrentOcc

- $P=p d f\left(X_{\text {CurrentHr }}\right.$, ValidOcc $)$

- $P$ Norm $=P$ normalized

- CurrentOcc = Randomly draw an occupancy from ValidOcc using probabilities PNorm

- Otherwise

- CurrentOcc $=$ All rooms are empty

\subsubsection{Assumptions}

There are several assumptions that are made to increase efficiency and to account for physical restrictions. When determining valid occupancies, the list of possible occupancies is not exhaustive. We assume that a maximum of two people can move through the lab, office, and elevator doorways. For slightly larger hallway entrances, we assume a maximum of three people can pass over a transition boundary. These assumptions are based on the maximum transitions observed in the ground truth data. The last assumption made is that people are not moving through multiple doorways concurrently. This is done to reduce the total number of possible occupancies that need to be examined and the time required to run simulations. The data shows that transitions rarely occur concurrently. Out of the 48 hours of ground truth data, only $4.68 \%$ of transitions occurred concurrently.

\subsection{Agent Based Model}

An agent-based model of part of the 2nd floor of the Science and Engineering building at UC Merced has been developed to simulate people dynamics. The simulation model we have employed is analogous to the cellular automata models [7]. The model takes into account the building geometry and simulates each individual's movement, and is able to provide quantitative output such as occupancy and congestion lev- 
els at any location and at any time period. In order to simulate each individual's movement, agent decisions must be modeled on several levels, such as itinerary, path choice, and walking behavior [1]. Itinerary decision determines the time of arrival and entrance, the number of stops, and the location and dwell time of each stop, for each individual. Path choice is the decision on which path to follow, given the individual's destination and a set of alternatives. Walking behavior is determined by factors such as average speed, average space taken by each individual, and conflict resolution rules at times when conflicts between agents arise.

In our current implementation, the spatial grid size has been set to be 2 by 2 feet cell (with discrete time step of 0.5 sec) and an agent follows a shortest path. Occupant itinerary has been calibrated using arrival time and entrance, number of stops, and location and dwell time of each stop from traffic data collected from web cameras located along the corridors of the S\&E building. Preliminary results indicate that the calibrated model-based predictions are reasonable when compared to raw measurements. The calibration procedure is described as follows.

In the first step, individual camera data were combined based on camera location, direction and sensor event time stamps, converting raw measurements into meaningful trajectories. Each trajectory has a time stamp indicating its initiating time, and zone identification numbers indicating its start and end location in the building. For example, if the camera data indicates that boundary 1 has an event at time t, boundary 2 has an event at time $\mathrm{t}+\Delta$, and $\Delta$ is within the typical time range used to go from boundary 1 to boundary 2 , then a trajectory can be formed by combining the two events indicating at time $t$, a person moves from zone $i$ to zone $j$ through boundary 1 and 2 . In the second step, a heuristic procedure was followed to assign trajectories to agents, from which agent itineraries were formed.
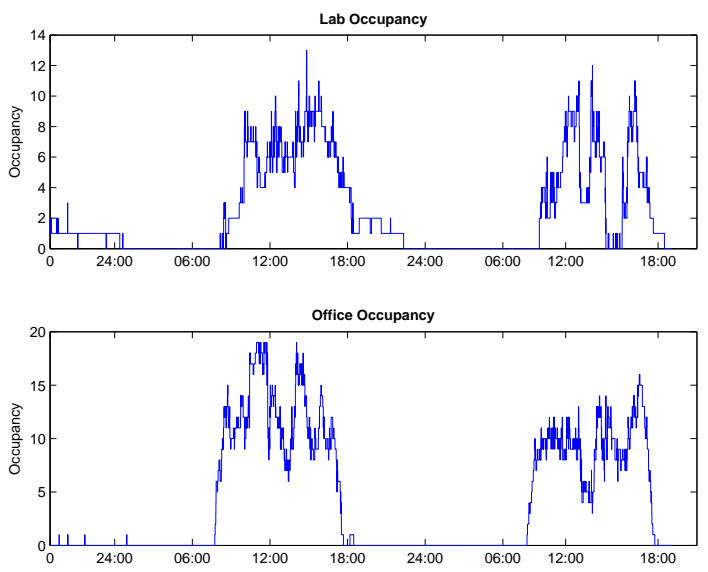

Figure 2. 48 hour ground truth occupancy data.

\section{Model Comparison}

In this section, we compare the performance of the MVGM and ABM, and examine the merits of each approach. The main statistics used for comparison are root mean squared error (RMSE) and normalized root mean squared error
(NRMSE). The RMSE is the average difference in occupancy for a given simulation.

\subsection{Initial Exploration}

As expected, regular patterns in the occupancy data are observable (refer Figure 2). The office plot shows the occupancy steadily increasing until just after noon. At around 14:00:00, people in the office leave for lunch and then return shortly after. By 18:00:00, the entire office is empty. One noticeable discrepancy can be observed when comparing peak office occupancy of each day. The first day shows a maximum office occupancy of 18 whereas the second day shows a maximum occupancy of only 13 . This is due to a large lunch meeting of six people on the first day. Typically the office staff is around 12 people. The lab plot shows a slightly different patterns but still is consistent with intuition. Students arrive in the morning, go to lunch, and then stay until late evening. Unlike the office staff, many students arrive mid-afternoon and leave much later.

\subsection{Multivariate Gaussian Model Results}

The MVGM was trained using the first 24 hours of data from the ground truth. The second 24 hours of ground truth was used as a testing set. For each model, 20 simulations were generated. Figures 3 shows a sample simulation generated by this model. From the plots, we can see that the simulation seems to capture the major events that occur during the day such as arriving for work and going to lunch. One noticeable difference is that simulations contain a fair amount of noise. This is most likely caused by the random sampling. Though each occupancy sampled is possible under the sampling rules, the rules do not prevent certain types of unlikely events. For example, it is possible under our current scheme for a person to enter the hallway through a particular door and then with fairly high probability immediately exit out of the same door. This "pacing" behavior seems to be the cause of this noise. This could potentially be corrected with additional sampling rules. On average, simulated lab occupancy differs from the test set occupancy by 3.462 people (RMSE) which is an average error of $28.8 \%$ (NRMSE). The simulationed office occupancy shows an average difference of 7.453 (NRMSE of 46.5\%). If we examine the combined total occupancy of both rooms, we find that the simulated total occupancy differs from the training set by 10.214 (NRMSE of $42.6 \%$ ).

\subsection{Agent Model Results}

The ABM was trained using the same 24 hour data set as the MVGM. To generate occupancy profile for lab and office, a day is divided into six time periods taking into account different behavior during different time of the day, e.g., early morning, lunch time and late afternoon. For each time period, empirical distributions of arrival time and duration in the lab/office were collected from the agent-based model. Based on the distributions, 20 simulation runs of the ABM were generated representing a variety of possible occupancy patterns of the lab and office. Figure 3 shows a sample simulation produced by the ABM. Like the MVGM, the ABM also seems to generate plausible simulations that capture the major shifts in occupancy. The simulated lab occupancy differs from the test set occupancy by 3.774 people (RMSE) 

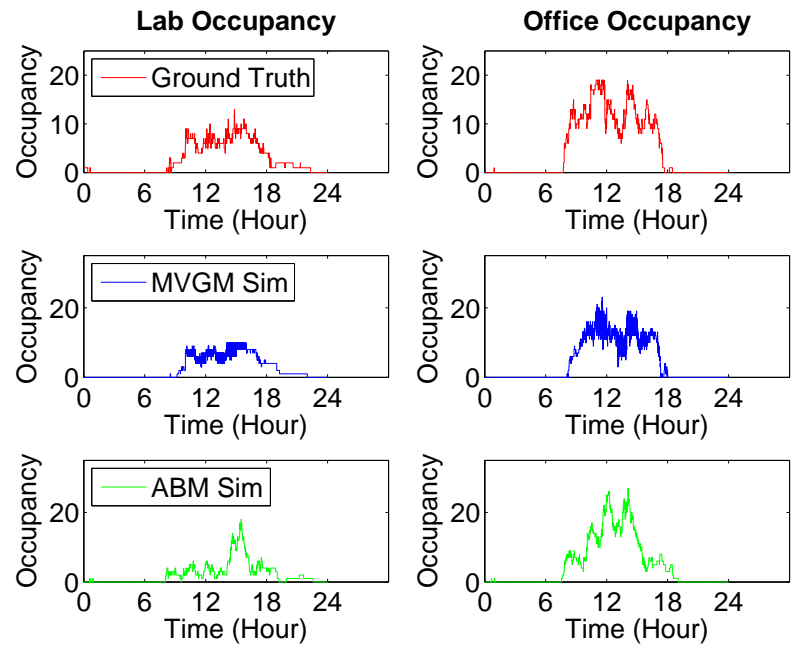

(a)

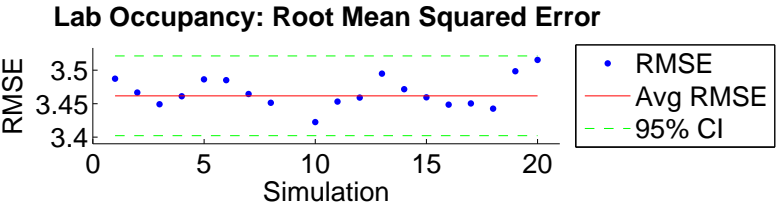

Office Occupancy: Root Mean Squared Error

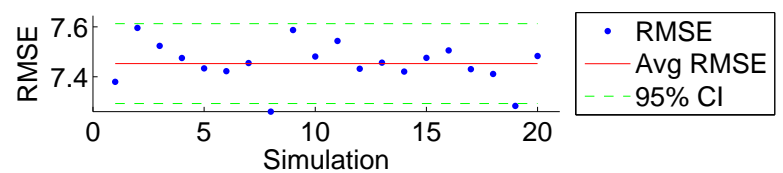

Total Occupancy: Root Mean Squared Error

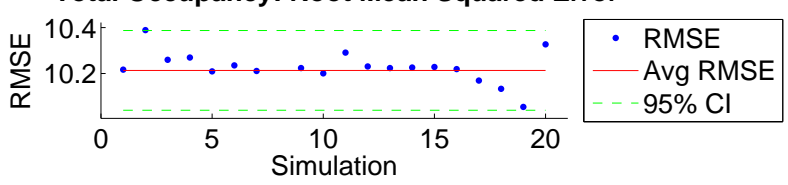

(b)

Figure 3. (a) Comparison of ground truth traces for lab and office areas with MVGM and ABM simulation traces. (b) Variation in RMSE along with 95\% confidence interval for MVGM simulation traces.

\begin{tabular}{r|cc|cc} 
& \multicolumn{2}{|c}{ MVGM } & \multicolumn{2}{c}{ ABM } \\
& RMSE & NRMSE & RMSE & NRMSE \\
\hline Lab & 3.462 & 0.2885 & 3.774 & 0.3145 \\
Office & 7.453 & 0.4658 & 7.577 & 0.4736 \\
Total & 10.214 & 0.4256 & 10.395 & 0.4331
\end{tabular}

Table 1. This table shows the average RMSE and NRMSE for simulations generated by the MVGM and the ABM.

\begin{tabular}{r|cc} 
& RMSE & NRMSE \\
\hline Lab & 3.370 & $19.4 \%$ \\
Office & 3.107 & $28.1 \%$ \\
Total & 5.688 & $23.7 \%$
\end{tabular}

Table 2. This table shows the RMSE and NRMSE when comparing the 24 hour testing and training datasets.

which is an average error of $31.5 \%$ (NRMSE). The simulationed office occupancy shows an average difference of 7.577 (NRMSE of $47.4 \%$ ). The simulated total occupancy differs from the testing set by 10.214 (NRMSE of 43.3\%).

\subsection{Comparison of Models}

With respect to RMSE and NRMSE, both models seem to have similar performance. Though both models seem to have a large amount of error, this error is actually reflecting the amount of occupancy variation that is possible between different days. If we compare the 24 hours testing set to the 24 hours training set, we find that the NRMSE for the lab, office and total occupancies are $28.1 \%, 19.4 \%$, and $23.4 \%$ respectively (refer Table 2). This shows that a significant amount of occupancy variation can be occur between days. One noticable difference between the simulations is the absence of noise in the ABM simulations. Since the ABM requires agents to follow a predefined path, this prevents people pacing between areas. In this respect, the ABM seems to capture the subtle occupancy variablility better than MVGM.
While both models seem to produce plausible simulations, each has its advantages and disadvantages. The ABM can be applied to other structures where an occupancy sensing infrastructure does not exist by training the $\mathrm{ABM}$ with data from another building with similar dynamics. This is not possible with the MVGM. The ABM is useful for building designers looking to maximize energy savings. The MVGM is more useful for real time prediction. Unlike the ABM which simulates occupancies offline, the MVGM can predict occupancy given the time of day and current occupancy by calculating the posterior probability given the current occupancy on all or part of the building.

\section{Optimal L-HVAC Strategies}

Traditionally, lighting, temperature, and ventilation (outside air - OA) control are based on schedules which rely on room usage assumptions. However, this assumption frequently overestimates the occupancy of spaces. On the other hand, if occupancy information is known, one could control lights, temperature, and ventilation levels to the appropriate level required, and set them back to minimum or off conditions when no occupancy is detected, resulting in energy savings. In this paper we illustrate the impact of ventilation control strategy on energy savings. Ventilation air is introduced into the building to avoid poor indoor air quality and is a function of the number of people in the building and the square footage. In absence of real-time occupancy information, OA amount is fixed based on maximum design occupancy assumption. Excess outdoor air has significant penalty on heating energy and depending on the outside temperature, even cooling energy. Thus varying the amount of air during nonfree cooling times to the minimum required to maintain acceptable indoor air quality has potential for saving energy.

An existing eQuest model [3] of Classroom and Office building (COB) on the UC Merced campus, developed during the building design stage, was used to understand the energy savings potential of using occupancy estimation for 
ventilation control. EQuest is an hourly whole building energy simulation tool used widely by the building community. It uses information on building configuration, schedules of building usage including people, lighting, plug loads, HVAC system configuration, expected cooling, heating and ventilation levels, and weather conditions to calculate the building energy consumption.

Two ventilation control strategies were simulated: base and new. In the base case, the OA quantity is based on maximum design occupancy, available during the occupied time ( $8 \mathrm{am} 10 \mathrm{pm})$. This quantity is fixed during the occupied time, irrespective of occupancy. The new ventilation strategy has OA quantities in all zones following the occupancy in the respective zones. All the strategies simulated consider the applicable codes. The daily actual occupancy levels over time for various HVAC zones in COB is estimated from available information on schedules for classrooms and an ABM for people movement in offices.

\subsection{Occupancy schedule for part of $\mathrm{COB}$}

The S\&E building has multitude of HVAC systems deployed for a variety of purposes including office spaces as well as clean room or laboratories. Due to the simplicity of the HVAC configuration in the COB building, and its primary usage for offices and classrooms with variable occupancies, the COB building was chosen for the energy savings estimation. Also, since energy models had previously been developed for the COB building, the goal was to take advantage of these models and examine potential energy savings for this building using simulated occupancy schdedules. Currently it is not possible to measure occupancies directly in the COB building. However, since the S\&E building has similar traffic patterns to the $\mathrm{COB}$, an $\mathrm{ABM}$ trained using data from the $\mathrm{S} \& \mathrm{E}$ building was developed to construct occupancies schedules for some portions of the COB building.

The occupancy modeling work for the UC Merced COB has been focused on the office section of second floor. Statistical distributions of parameters which define occupant itinerary; arrival time, dwell time, and number of stops, are extracted from the ABM from the S\&E building which is expected to have similar traffic. The information regarding different types of occupants that use the building (and their relative proportions) and occupancy level for whole office section, is derived from different types of rooms in the section and maximum occupancy expected in each room. Finally, by Monte Carlo sampling of the occupant itinerary parameters and averaging over different realizations of traffic patterns obtained from simulating the model, occupancy profile over the course of the day for each room is generated. This helps us assess the impact of using the time resolved occupancy distribution on energy savings.

\subsection{Energy Savings via Optimal Control of L- HVAC}

Results show that $5 \%$ of HVAC energy savings is possible compared to the current outside ventilation air control strategy and about 14\% HVAC energy savings is possible when compared with base-case outside air control strategy. Sensitivity calculations of energy savings to occupancy estimation errors and sensor bias were conducted. Results show that $20 \%$ occupancy estimation errors have negligible impact $(0.28 \%)$ on HVAC energy savings estimation of $14 \%$. A sensor bias of $20 \%$ results in $3 \%$ change in energy savings estimation.

\section{Conclusions}

In this work, we present the first steps in understanding dynamic occupancy levels and patterns in buildings, means by which they can be estimated, and the energy efficiency gains possible by utilizing actual facility usage information for building controls. As proof of concept, we deployed an 16-node wireless camera sensor network in a multi-function building to determine the occupancy resolution one can conceive of obtaining in a typical building. These results in turn were used to demonstrate an example of an occupancy-based energy control. The results suggest that, in many buildings and for many building uses, we will find that knowing the occupancy and usage patterns will result in significantly higher energy savings compared to strategies assuming fixed occupancy and usage patterns. Our future research directions involve doing online L-HVAC control using only a wireless camera sensor network to provide occupancy estimates for longer durations (days, months).

\section{Acknowledgments}

This work was supported in part by the Department of Energy and the California Energy Commission, and performed under U.S. Department of Energy Contract No. DE-AC0205CH11231 and the California Institute for Energy and the Environment under contract No. MUC-09-03.

\section{References}

[1] G. A. Bierlaire, M and M. Webers. Behavioral dynamics for pedestrians. In 10th International Conference on Travel Behavior Research, Aug. 2003.

[2] M. J. Brandemuehl and J. E. Braun. The impact of demand-controlled and economizer ventilation strategies on energy use in buildings. ASHRAE Transactions, 105(2), 1999.

[3] eQuest Building Energy Analysis Tool. http://www.doe2.com/.

[4] A. Kamthe, L. Jiang, M. Dudys, and A. Cerpa. SCOPES: Smart Cameras Object Position Estimation System. In In the Proceedings of the 6th European Conference on Wireless Sensor Networks(EWSN 2009), pages 279-295, Cork, Ireland, Feb. 2009. Springer-Verlag.

[5] F. W. Payne. Energy management control system handbook. Fairmont Press, 1984.

[6] S. P. Meyn, A. Surana, Y. Lin, S. M. Oggianu, S. Narayanan, T. Frewen. A sensor-utility-network method for estimation of occupancy distribution in buildings. In Proceedings of IEEE Conference on Decision and Control, Shanghai, China, 2009.

[7] A. Schadschneider. Pedestrian and Evacuation Dynamics, chapter Cellular automaton approach to pedestrian dynamics theory, pages 75-86. Springer, 2002. 
To be Published in Proceedings of “ACEEE 2010 Summer Study for Building Energy Efficiency” August, 2010.

\title{
Systems Approach to Energy Efficient Building Operation: Case Studies
} and Lessons Learned in a University Campus

\author{
Satish Narayanan, United Technologies Research Center \\ Michael G. Apte, Philip Haves, Mary Ann Piette, Lawrence Berkeley National Laboratory \\ John Elliott, University of California Merced
}

\begin{abstract}
This paper reviews findings from research conducted at a university campus to develop a robust systems approach to monitor and continually optimize building energy performance. The field analysis, comprising three projects, included detailed monitoring, model-based analysis of system energy performance, and implementation of optimized control strategies for both districtand building-scale systems. One project used models of the central cooling plant and campus building loads, and weather forecasts to analyze and optimize the energy performance of a district cooling system, comprising chillers, pumps and a thermal energy storage system. Fullscale implementation of policies devised with a model predictive control approach produced energy savings of about 5\%, while demonstrating that the heuristic policies implemented by the operators were close to optimal during peak cooling season and loads. Research was also conducted to evaluate whole building monitoring and control methods. A second project performed in a campus building combined sub-metered end-use data, performance benchmarks, energy simulations and thermal load estimators to create a web-based energy performance visualization tool prototype. This tool provides actionable energy usage information to aid in facility operation and to enable performance improvement. In a third project, an alternative to demand controlled ventilation enabled by direct measurements of building occupancy levels was assessed. Simulations were used to show 5-15\% reduction in building HVAC system energy usage when using estimates of actual occupancy levels.
\end{abstract}

\section{Introduction}

One of the problems that contribute to higher than expected energy use in commercial buildings is the lack of actionable data and analysis tools to link control strategies, operations and energy use in the built environment. Conventional building operations are subject to several problems. Monitoring and diagnostics systems rely on a variety of measured data sources to gain insights to the actual building performance, with limited understanding of the information uncertainty and lacking a simple and actionable operator interface. Such limitations result in the inability to diagnose and have corrective actions when the building or its systems are not behaving as expected. Use of fixed schedules and equipment set points (based on equipment performance optimization) limit the ability to achieve overall energy use reduction. The projects described here aimed to address such problems and assess approaches to minimize energy waste and optimize operations in commercial buildings. The following describes three closely linked projects conducted at the University of California, Merced (UC Merced) to implement and evaluate technologies at a district and building scale for enhanced facility operations and energy performance through use of improved control and visualization tools, enabled by data and system-level models. The efforts take advantage of the state-of-the-art monitoring systems deployed at the UC Merced campus. A strong commitment to energy efficient building design 
and operation as well as sustainability have resulted in deployment of a program to design buildings that consume half the energy and peak demand of other university buildings in California (Brown 2002) and numerous LEED new construction green building certifications (6 LEED Gold and 1 LEED Silver to date). The campus uses an energy management and control system (EMCS) through which energy and equipment performance data can be remotely accessed. This includes a comprehensive monitoring and metering system in which over 10,000 points are tracked across $900,000 \mathrm{ft}^{2}$ of built space (see Granderson et al. 2009).

\section{Research and Development Goals and Objectives}

Model Predictive Control of Chilled Water Plant System. The overall goal was to assess the feasibility and energy performance benefits of optimal control of the central cooling plant. The objective was to evaluate the feasibility of and to demonstrate the energy savings potential of model predictive control (MPC) for set-point optimization and scheduling of a district cooling system with thermal storage serving the UC Merced campus. Energy savings for the demonstration were expected to be around $10 \%$ based on published simulation and experimental studies of MPC applied to chilled water plant with storage (Flake 1998; Henze et al. 2005).

Energy Performance Visualization System. The project objective was to demonstrate real-time energy performance visualization capability for the UC Merced Classroom and Office Building (COB). An approach which combined sensor data with building thermal and energy performance models was developed. The project aimed to demonstrate the following advances: (i) techniques to process data sources of loads and environmental variables with quality metrics for comparison with performance benchmarks; (ii) whole building energy simulations suitable for real-time use; and (iii) a prototype of a real-time performance monitoring system that integrates these elements to visualize actionable information. When implemented with the full capability, this technology will enable building data to be easily accessible by a broader community for research and provide operators with a sufficiently detailed and transparent understanding of facility operation to track energy usage against performance benchmarks and diagnose operational issues.

Occupancy-Based Energy Management System. The objective of this project was to investigate opportunities to reduce energy use in a UC Merced building by adjusting the HVAC system operation, based on real-time knowledge of actual building occupancy and contrast with traditional $\mathrm{CO}_{2}$ sensor-based demand controlled ventilation strategies. The research focused on the Science and Engineering (SE) building and COB and included analyses to: (1) determine the building control options and associated energy benefits for a given level of detail about occupancy (e.g., spatial distribution and temporal resolution), and (2) characterize the sensor hardware and assess models needed to directly estimate building occupancy.

\section{Technical Approach and Methodologies}

Model Predictive Control. MPC offers energy saving potential in large buildings and systems for which response to external disturbances or control inputs is slow, i.e. on the order of hours. MPC effectively provides a means to optimize systems dynamically to take advantage of building utilization, weather patterns, and utility rate structures. An MPC scheme was developed and tested for the UC Merced campus chilled water system. The control algorithms were implemented in MATLAB, with readily available tool boxes for rapid development and 
performance assessment. Dynamic models of the chilled water piping system and of the buildings were developed in the Modelica language (Wetter 2009) and used as the basis of the MATLAB models and lookup tables for use in the control policy design.

The main components of the campus chilled water system are schematically depicted in Figure 1. The chilled water plant consists of three 1,200 ton chillers, a cooling tower, a 2,000,000 gallon thermal energy storage (TES) tank, a primary chilled water distribution system and secondary distribution loops serving each building, managed by a single building automation system. The existing controls maintain $39^{\circ} \mathrm{F}$ leaving water temperature and the chillers are sequenced manually to maintain each chiller as close to full load as possible while producing sufficient stored chilled water for the following day.

Detailed descriptions of the individual components comprising the chilled water plant are presented in Haves et al. (2010) and in Ma et al. (2009, 2010). Two TES tank models were developed to predict the total stored cooling capacity, the temperature of the water supplied to the campus, and the temperature of the water returned to the chiller. In the more detailed model, the temperature profile in the tank is modeled by discretizing the tank into a number of layers. For online optimization, a low-order model was developed in which the cool and warm water are treated as lumped masses and the thermocline between the warm and cool water is treated as a moving boundary, thus requiring only three dynamic states, i.e. the position of the thermocline and the temperature of each lumped mass. The control design includes a simple, lumped parameter model that predicts the total campus cooling load based on the ambient temperature, the cloud cover, the time of day and the day of the year.

Figure 1: Schematic diagram of UC Merced chilled water system.

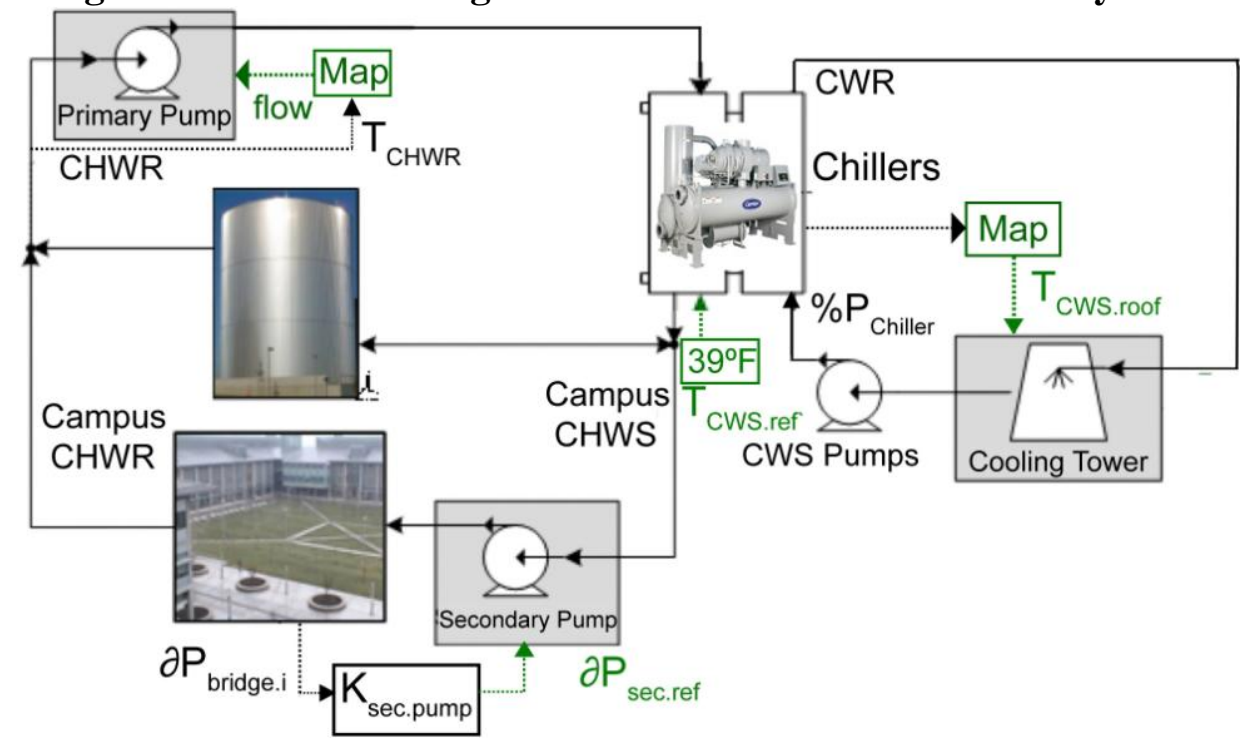

The aggregate campus chilled water flow rate and return temperature are predicted by a single cooling coil model that represents the combined effect of all the cooling coils on the campus. The model parameters are identified from measured data. Figure 2 shows a comparison of the predicted and measured cooling load and return water temperature. Based on building load and weather forecasts, optimal control policies were created to adjust chilled water plant setpoints including leaving water temperature, cooling tower return temperature, chiller staging, and the volume of chilled water stored in the tank. A cost function that includes energy consumption and peak electrical demand over a 24-72 hour prediction horizon was formulated and solved 
using a one hour time-step (Haves et al. 2010, Ma et al. 2009, 2010).

Figure 2: Fall 2009 (a) Campus cooling load, (b) Campus return temperature versus load.
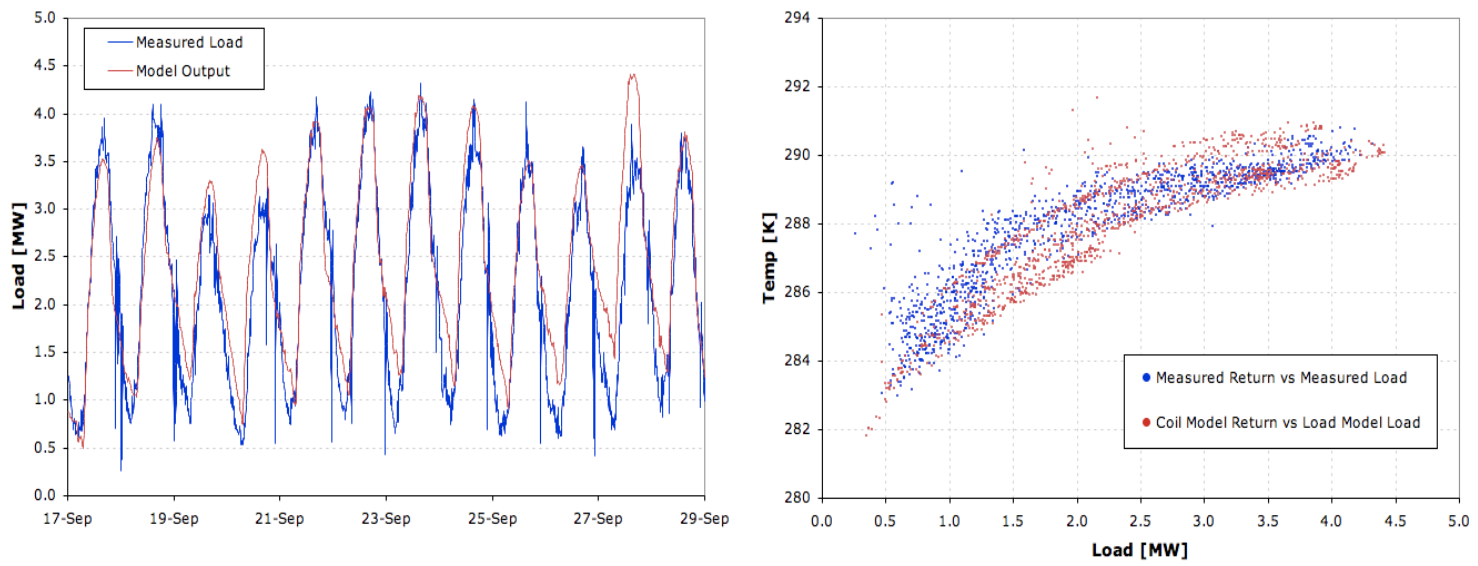

Energy Performance Visualization System. The visualization system aimed to provide concise information on building system conditions, utilization, operation, and energy performance broken down by end use. A prototype was developed and evaluated for the COB at UC Merced. The main components of the visualization prototype are depicted in Figure 3.

Figure 3: Performance visualization tool framework and key elements.

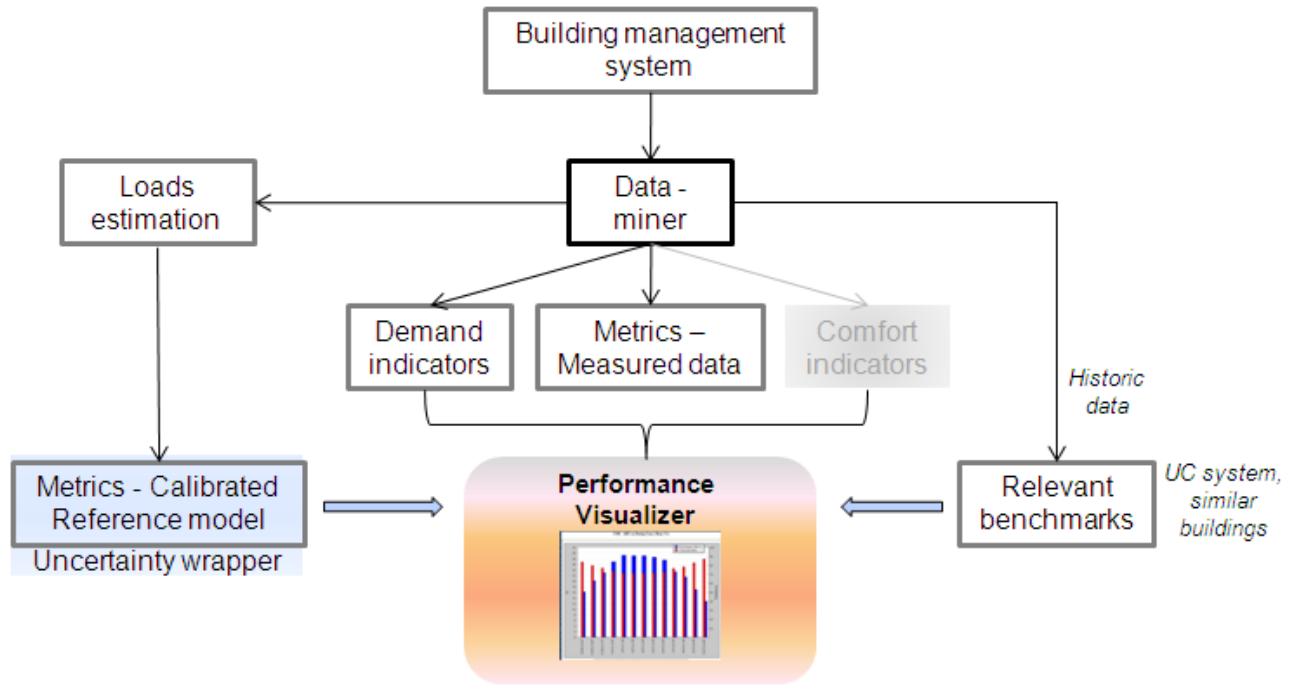

Building management system database. Data is recorded by the building management system (BMS) for control, stored locally for a short period of time, and is then archived to a database. The trending data is transferred to a dedicated server that is queried from the visualization tool.

Data miner. The data miner was developed with a web-based GUI that processes the archived data for use by the visualization tool and to make it easy for users to access and manipulate both the archived data and the outputs of the building model (described later). The website serves as the interface through which users query the SQL database. Through this site users have the ability to plot and download datasets of their choosing, i.e. the specific operational variables and 
energy use information and required time intervals (daily, monthly or annual). The ability to modify basic plotting features, such as the choice of daily averages and edge plots, allows for customized visualization of the data. Importantly, as the pre-processing tool for trend data, the data miner serves as an inspection point for data quality.

Performance metrics. Performance metrics consolidate the vast amount of energy data, usually trended as part of the BMS, into standardized quantities for easier and quicker understanding of the building operation. Performance metrics based on prior work (e.g., Gillespie et. al. 2007), for three categories were developed - whole-building (e.g., total energy consumption, cost, carbon emissions), end-use energy, and operational efficiency (e.g. cooling plant, heating, fan efficiencies). The metrics were calculated from the UC Merced metered trending data, and were tailored based on the campus facility manager's feedback. Table 1 outlines the data needed for each of the metrics at the whole building level and the procedure for calculating the metric (for details see Apte et al. 2010). The performance metric value depends on the demand indicators including heating and cooling degree-days and percent of hours the HVAC system is in the occupied mode. Comfort indicators to be implemented will include the space heating and cooling set points. There are usually several periods when the data is either not logged or logged incorrectly. Decisions on whether data is incorrect are made based on domain expertise using standard imputation techniques. For all metrics, the percent of missing values is reported, giving the user a confidence rating, and providing transparency that is lacking in typical EMCS.

\begin{tabular}{|c|c|c|c|}
\hline Metric & Unit & Data & Calculation $^{2}$ \\
\hline $\begin{array}{l}\text { Total } \\
\text { electricity } \\
\text { consumption }\end{array}$ & $\mathrm{kWh} / \mathrm{yr} / \mathrm{gsf}$ & $\begin{array}{l}\text { - } \mathrm{kW} \text { data at every time step, for a } \\
\text { whole year } \\
\text { - Total gross sq.ft. (incl. the wall) }\end{array}$ & Sum (kW data at every time step) \\
\hline $\begin{array}{l}\text { Electricity } \\
\text { demand }\end{array}$ & $\mathrm{kW} / \mathrm{gsf}$ & Same as above & Max (kW data at every time step) \\
\hline $\begin{array}{l}\text { Total gas } \\
\text { consumption }\end{array}$ & therms/gsf-yr & $\begin{array}{l}\text { - Therms/hr hot water data at } \\
\text { every time step, for a whole year } \\
\text { - Total gross sq.ft. (incl. the wall) } \\
\text { - Central boiler plant efficiency }\end{array}$ & $\begin{array}{l}\text { Sum (therms/hr gas consumption data at } \\
\text { every time step) }\end{array}$ \\
\hline Gas demand & therms/hr-gsf-yr & Same as above & $\begin{array}{l}\text { Max (therms/hr gas consumption at every } \\
\text { time step) }\end{array}$ \\
\hline
\end{tabular}

The points to trend in a BMS are typically decided based on operational requirements, and not from energy performance standpoint. For the COB, the trended dataset is rich and maps almost one-to-one to the performance metrics outlined here. The one case where it does not map directly is for interior lighting power consumption because other electric circuits (e.g. outdoor lighting, certain pumps) are mixed into the lighting power metering panel.

Performance benchmarks. Historic baseline data and whole-building reference models allow for comparison of current performance with benchmarks at system, sub-system, component (e.g., fan coil), and component parameter level (e.g., fan coil temperature, water flows). For this project, benchmarks for annual consumption based on comparable buildings (Brown 2002) are used (see

\footnotetext{
${ }^{1}$ See Apte et al. 2010 for more details on measurements and on end use energy metrics

${ }^{2}$ Calculated quantities have the product of (the number of time steps in one hour) $\mathrm{x}$ (building gsf) in the denominator
} 
Table 2). For some metrics, design intent/standards are used as benchmarks. Benchmarks can also be derived from whole-building and end-use stock models such as LBNL's EnergyIQ (http://energyiq.lbl.gov/SupportPages/EIQ-about.html), drawing upon analysis of the California End Use Survey (Mathew et al. 2008). For COB, historic data are available to generate same building historic baseline data for every metric and at any time interval. The comparable building benchmarks are based on 1999 UC/CSU campus benchmarks (Brown 2002), and are calculated using regression models, accounting for space usage (e.g., percent office or laboratory space) and climate. Another set of benchmarks are goals used in energy efficient designs. For example, 1 $\mathrm{cfm} / \mathrm{ft}^{2}$ is a typical metric for installed fan flows. This number tends to be lower $(\sim 0.8 \mathrm{cfm} / \mathrm{sq} . \mathrm{ft}$.) for more efficient designs. A careful presentation of $\mathrm{COB}$ benchmarks, targets, and actual performance can also be found in a New Buildings Institute study ${ }^{3}$.

Table 2: Comparable building benchmarks used for COB

\begin{tabular}{|l|l|l|l|}
\hline & Units & Target & Comment \\
\hline Max Electric Demand & $\mathrm{W} / \mathrm{gsf}$ & 3.65 & \multirow{2}{*}{$\begin{array}{l}\text { Includes allocated cooling plant, building } \\
\text { exterior lights, and allocated campus road lights }\end{array}$} \\
\cline { 1 - 2 } Annual Electric Use & $\mathrm{kWh} / \mathrm{yr} / \mathrm{gsf}$ & 15.1 & \multirow{2}{*}{ Includes hot water and heating } \\
\hline Max Gas Demand & $\mathrm{Th} / \mathrm{hr} / \mathrm{kgsf}$ & 0.12 & \\
\hline Annual Gas Use & $\mathrm{Th} / \mathrm{yr} / \mathrm{gsf}$ & 0.2 & \\
\hline Max Cooling & Tons/kgfs & 2.03 & \\
\hline
\end{tabular}

EnergyPlus simulation model. With the advent of the LEED rating system the use of whole building energy simulations for design is becoming common. These models (appropriately calibrated) can be used during operation, to track expected performance, and to understand subsystem behavior to isolate operational problems and identify means to improve building energy performance. A simulation model for the COB was created using EnergyPlus version 4.0.0.024 (see Figure 4). Details of the model are provided in Apte et al. (2010), and its calibration is still in progress. Models for the building surfaces (i.e. walls, insulation, overhangs and glazing) were incorporated. Key internal loads modeled were people, lights, and plug-load equipment and were specified for each model zone by a maximum value (people and lights) or a per floor area value (equipment). Occupancy level and lighting power information was obtained from architectural drawings. Weather files for the years 2008 and 2009 were compiled from several sources (UC Merced: air temperature and wind speed; direct normal radiation: California Department of Water, San Luis Reservoir site; relative humidity for 2008 Fresno International and Merced Municipal Airports). Electrical consumption by lighting, equipment, and fans was disaggregated into categories corresponding to the electric sub-meters installed in the COB. Figure 5 shows preliminary comparisons of simulation results and sub-metered data, showing discrepancies from factors such as improperly matched schedules (Apte et al. 2010).

Indoor thermal load estimation. Insights into the dynamics of building loads can help understand and optimize building energy performance. When available, sensors within the terminal units and indoor environment can provide useful information, but they can be grossly inaccurate when estimating loads over extended periods of time because of accumulating errors. An approach to estimate internal loads combining simple thermal network models (3R2C) with real-time data

\footnotetext{
${ }^{3}$ http://www.newbuildings.org/sites/default/files/Case_Study_UCM-COB.pdf
} 
from the BMS was implemented. The estimated internal loads were compared to measured data from the COB to ensure consistency (O'Neill et al. 2010). The internal load was estimated in a lumped form including internal lighting, equipment, people, infiltration and inter-zone mixing. The estimation captures the daily (daytime vs. nighttime) and weekly (weekday vs. weekend) variation for the loads and can shed light on anomalies in energy performance or operations.

Figure 4: Model representation of COB showing glazing and overhangs.

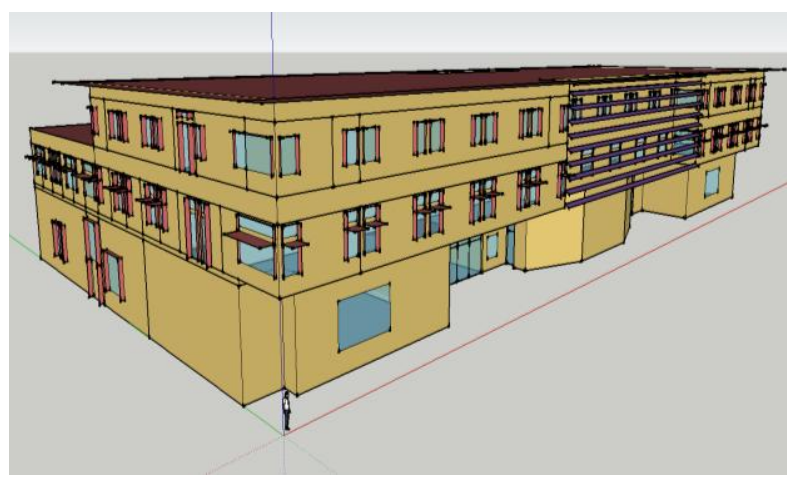

Figure 5: EnergyPlus predictions (solid) compared to measured (dashed) building electric consumption for Sep. 2009.

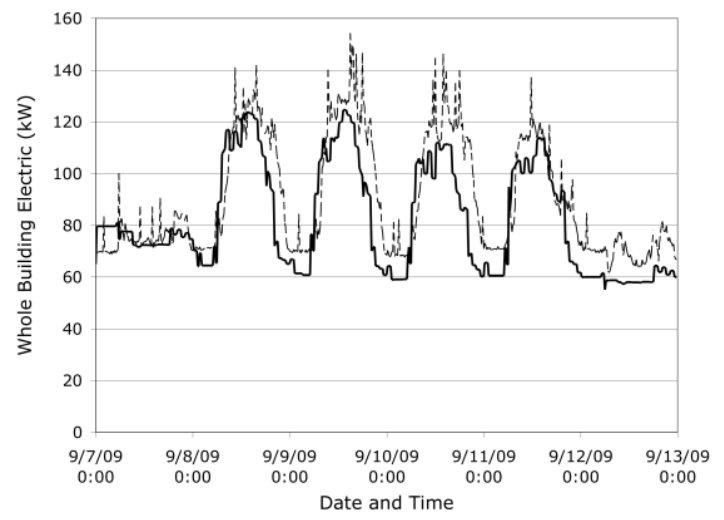

Performance visualizer. The performance visualizer provides an integrated environment and interface to display the metric values for the measured data and their historic values, the benchmarks, zonal loads where appropriate, and the reference EnergyPlus model side by side. The metrics can be visualized at yearly, monthly, weekly as well as daily intervals. In addition, for a given metric, the individual data points and demand indicators can also be displayed.

Occupancy-Based Energy Management System. The experiments to determine the feasibility of directly measuring or estimating the number and location of occupants in a building using a wireless network of low-power, low-resolution cameras were conducted in the SE building. The energy-savings potential from knowing the distribution of occupants was evaluated for the COB. An existing eQUEST model of $\mathrm{COB}$, developed during building design (Taylor engineering 2002), was analyzed to evaluate the energy savings potential of using direct occupancy estimation for ventilation control. The details of deploying a network of wireless camera sensors in the SE building are discussed in Erickson et al. 2009. When an occupant crosses key transition points (see Figure 6), the cameras capture and process the images to determine actual occupancy count. The resulting traces (see Figure 6) were used to train occupancy models (see Erickson et al., 2009). A combination of prior knowledge on building usage and models of traffic patterns in SE building faculty and graduate student offices and public areas were used to generate occupancy patterns for the COB in similar areas (where occupancy sensors were not available). Available schedules for different days were used for classrooms, simulating the use of error-free $\mathrm{CO}_{2}$ sensors in the $\mathrm{COB}$. The approach was as follows: (i) generate occupancy schedules for use in simulation environment; (ii) adjust control setpoints (temperature and ventilation levels in individual zones) based on occupancy level; (iii) use eQUEST model to predict energy consumption with control strategies. For design, occupancy was described by one class schedule and one office schedule for all seasons and classroom and office zones, respectively. To estimate the savings due to control based on direct measurement of occupancy, schedules are updated each classroom has a unique schedule. The classroom schedule varies, based on the day of the 
week, season, and vacation. Each office zone has a unique schedule, generated from the occupancy movement model (Erickson et al. 2009). The occupied time is assumed to be from 7AM to 9PM. The minimum flow ensured in spaces is $40 \%$ in classrooms, auditoriums, and conference rooms and $30 \%$ in offices. Literature-based estimates on demand controlled ventilation (employing $\mathrm{CO}_{2}$ sensors in every zone) suggested the potential for reducing HVAC energy consumption by 10-20\% in a typical office building (Emmerich and Persily 2001).

Figure 6: Wireless camera sensor network deployed in second floor of SE building (top). Typically observed occupancy patterns in lab and office spaces in SE building (bottom).
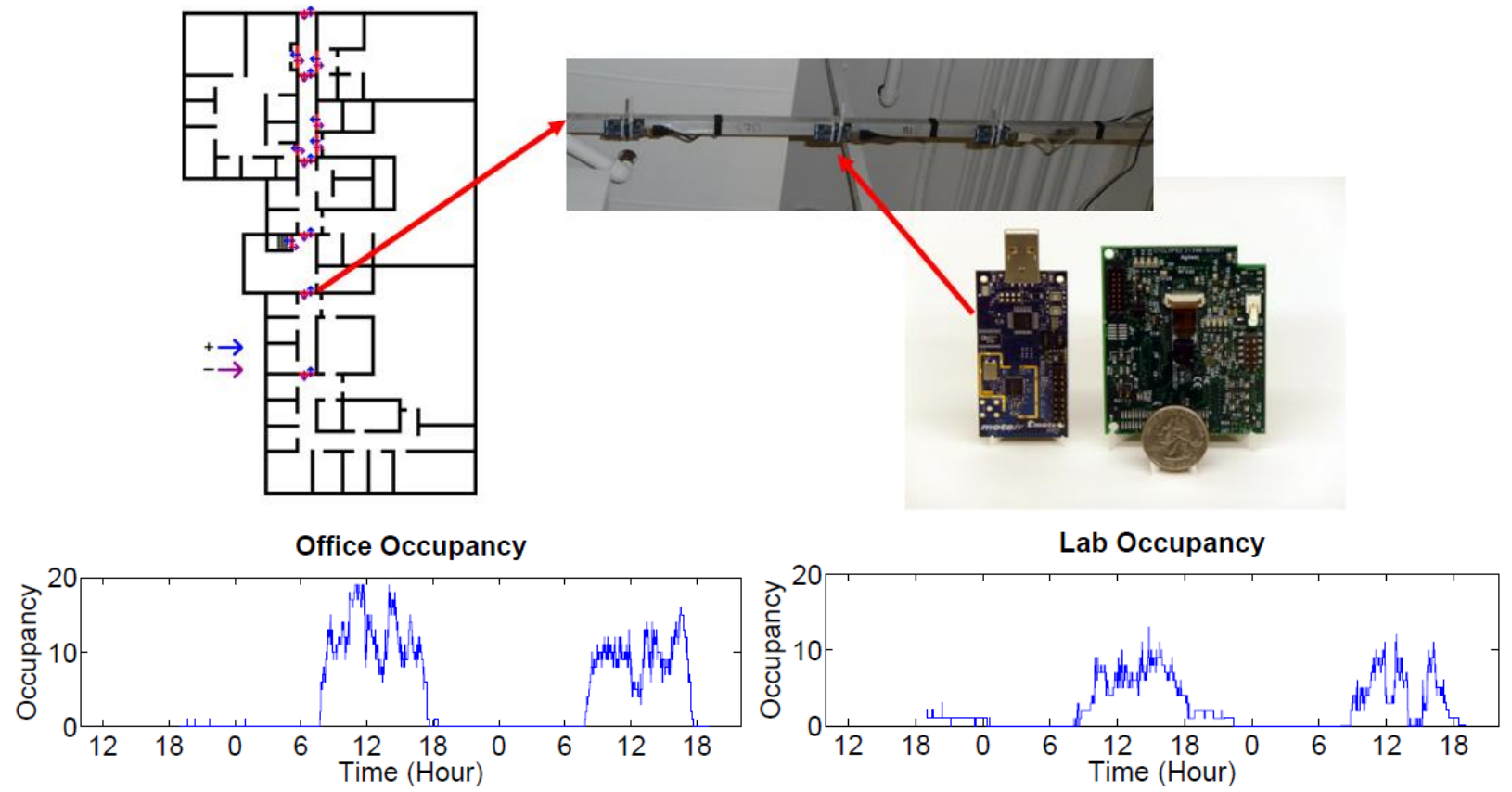

\section{Summary of Results}

MPC for Chilled Water Plant. Opportunities to optimize the chilled water plant runtimes were identified to take advantage of ambient conditions and eliminate overcharging of the chilled water tank. Two MPC experiments were carried out with the central cooling plant. The first was a week-long test in June 2009 during the summer/peak cooling season. Various algorithm and modeling bugs were found during this test, and performance improvements from the MPC implementation were not evident (Haves et al. 2010). Suboptimal choice of charging window length for the algorithm affected the overall COP adversely. However, an increase in system $\mathrm{COP}$ by increasing the standard condenser water set-point (CWS) range from $57-60^{\circ} \mathrm{F}$ to $65-66^{\circ} \mathrm{F}$ was learned as a useful policy modification. Regression analysis suggests the COP improvement potential for the CWS change is approximately $1.5 \%$, although this was difficult to confirm due to the multiple changes that occurred simultaneously. A second MPC experiment was conducted in October 2009; the cooling load was much lower than during the summer. The incremental energy savings relative to the original manually implemented policy were $4.6 \% \pm 2.4 \%$. A simplified tool with rules derived from the above experiments is now being implemented at the UC Merced central plant. The campus load and plant models developed have also proved to be a useful commissioning tool for facility operation. For instance, it was determined that the CHWS 
set-point and the chilled water flow rate can be used to limit the chiller loading to prevent chiller surging. Inconsistencies in central and buildings-level return temperature data for campus load modeling led to the identification of a malfunctioning flow rate sensor in one of the buildings, which caused higher demands for chilled water and reduced chilled water return temperatures. The testing process also led to identification of simple modifications to the heuristic control policy currently used by the operators. It was found that operating the chillers near full load was a key factor in maximizing system efficiency, leading to the recommendation to operate a single chiller (at near peak load) in off-peak regimes (e.g. transition/shoulder seasons).

Figure 7: Comparison of benchmark, measured data, and reference model for whole building fuel usage and end-use performance metrics.
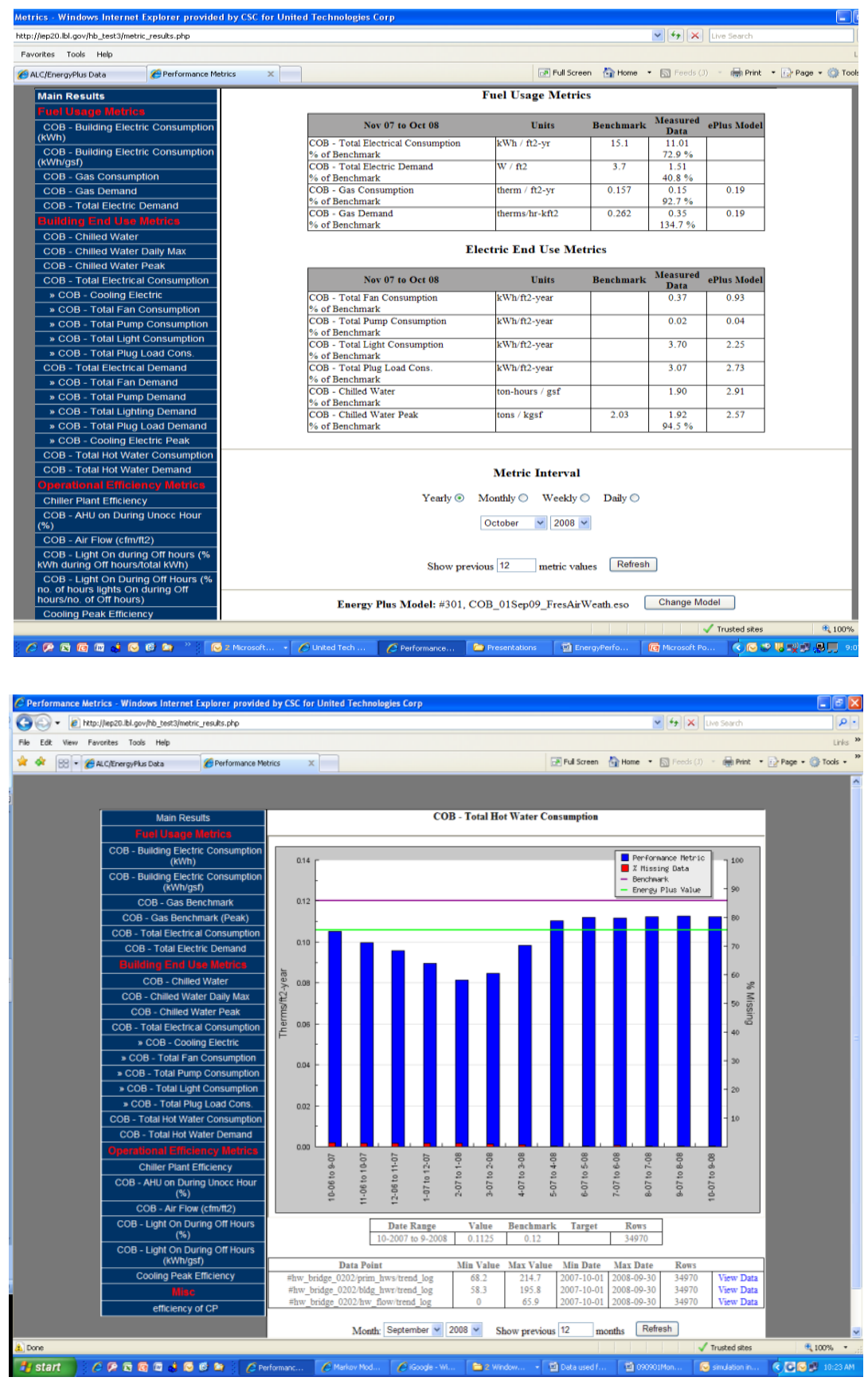
Energy Performance Visualization for COB. The performance visualizer displays metrics for measured data (including historic values), benchmarks, and a reference model side by side. For a given metric, the individual data points and demand indicators can also be visualized. Screenshots from the prototype are shown in Figure 7. The prototype supports performance tracking, and to some extent, the localization of performance degradation and faults to a subsystem/parameter level. This is enabled by time-series charts for the metrics that compare measured performance with benchmarks, historical same-building data, and metrics from a calibrated, reference whole building EnergyPlus model. The visualizer enables correlation between variances in energy performance measurements or dynamic indoor load estimates and relevant equipment operational variables. In one instance, a problem with secondary pump control for COB hot water delivery that was responsible for a temporary $36 \%$ increase in whole building heating energy use was identified and repaired.

The Occupancy-Based Energy Management System. Energy savings analysis from the use of occupancy-based controls was conducted for the COB. Three ventilation control strategies were simulated. In the base case, the outside air (OA) quantity is set based on maximum design occupancy. This quantity is fixed during occupied times, irrespective of the occupancy level, and commonly implemented. The COB eQUEST model was used to establish baseline energy consumption. A 4-5\% reduction of the annual whole building energy consumption was estimated compared to the base control strategy and only a marginal improvement of $\sim 1 \%$ from the current control strategy was observed (which utilizes $\mathrm{CO}_{2}$ sensor-based demand controlled ventilation for two-third's of the space); applicable ventilation code requirements (ASHRAE 62.12007 and Title 24) were ensured. This translates to an HVAC annual energy consumption reduction of about $14 \%$ using actual occupancy estimates and a 4\% energy use reduction when compared to current control strategy (Table 3). Note that the current demand controlled ventilation strategy simulated is assumed to be free of sensor uncertainties, which can be up to $20 \%$ for $\mathrm{CO}_{2}$ sensors.

Table 3: Saving calculations based on ASHRAE 62.1 2007 ventilation requirements

\begin{tabular}{|l|c|c|c|c|c|c|c|c|c|}
\hline & Heating & Cooling & $\begin{array}{c}\text { Heat } \\
\text { Reject }\end{array}$ & Pumps & Fan & HVAC & $\begin{array}{c}\% \\
\text { Savings } \\
\text { HVAC }\end{array}$ & $\begin{array}{c}\% \\
\text { Total }\end{array}$ & $\begin{array}{c}\text { Savings } \\
\text { Total }\end{array}$ \\
\hline Base Control Strategy & 335 & 252 & 5 & 150 & 128 & 870 & - & 2578 & - \\
\hline Current Control Strateg & 327 & 214 & 4 & 141 & 101 & 786 & $10 \%$ & 2494 & $3.3 \%$ \\
\hline New Control Strategy & 293 & 212 & 4 & 140 & 101 & 750 & $14 \%$ & 2457 & $4.7 \%$ \\
\hline
\end{tabular}

\section{Concluding Remarks}

The UC Merced research projects explored new methods to combine measurements, simulation models, control strategies, and information feedback to improve facility operation and reduce energy consumption in buildings. The cooling plant optimization project showed that dynamic system models could be used to identify critical control variables (from an energy performance standpoint), guide facility operation, and produce a predictive controller that reduces energy use. Full-scale implementation of control policies based on model predictive control demonstrated that the heuristic policies implemented by the operators were quite close to optimal; policies based on model predictive control produced energy savings of 5\%. The methodology is extensible to air-side HVAC systems and to building hydronic systems where variable speed technologies are becoming prevalent and robust, multivariable control methods 
are lacking. The projects extended the use of data into new analysis platforms for direct use by facility operators. The flexible and transparent web-based visualization prototype illustrates that comparative performance metrics are an effective way to understand the energy and operational performance of the building compared to current methods of data trending. With the visualization prototype providing comparison of performance metrics to previous years, building models, and benchmarks, the facility manager can assess the energy and cost savings of a particular action with relative certainty; a traditional BMS may not store data for the length of time necessary to provide such insight, nor does it provide relevant benchmarks or models to show how the building is expected to perform. The prototype comprising measured data and benchmarks is now being updated with new metrics and implemented operationally at UC Merced. It is anticipated that the value of this tool will become more apparent as various building systems age and require commissioning. It has been recognized that building energy consumption and electricity demand can be reduced by $10-15 \%$ when actionable energy usage information is provided to facility managers and operators (Mills and Mathew 2009) and the visualization prototype developed here is the first step in enabling this. Preliminary results from the occupancy-based energy management study revealed incremental benefits over conventional demand controlled ventilation strategies, indicating energy-savings potential arising from setting outside air ventilation based on measurements of the actual number of building occupants. Savings are anticipated to be higher in buildings where an extensive $\mathrm{CO}_{2}$ sensor network (such as in the $\mathrm{COB}$ ) may not be available.

\section{Acknowledgements}

The authors are grateful to a large team of researchers from LBNL, UTRC, UC Merced, UC Berkeley and UC Santa Barbara that were integral to the projects reviewed here. They include Andrzej Banaszuk, Pam Berkeley, Doug Black, Francesco Borelli, Scott Bortoff, Rohini Brahme, Brian Coffey, Al Cerpa, Varick Erickson, Jessica Granderson, Ankur Kamthe, Yiqing Lin, Yudong Ma, Igor Mezić, Zheng O’Neill, Eva Sevilla, Michael Sohn, Michael Spears, Amit Surana and Michael Wetter. This work was supported by the Assistant Secretary for Energy Efficiency and Renewable Energy, Office of Building Technology, State and Community Programs of the U.S. Department of Energy under Contract No. DE-AC02-05CH11231 and by the California Energy Commission PIER Buildings program through the California Institute for Energy and the Environment (CIEE). Authors are also grateful to Karl Brown (CIEE) for his support and guidance during the projects.

\section{References}

Apte, M., Sohn, M., Piette, M-A., Berkeley, P., Black, D., Price, P., Najafi, M., Narayanan, S., Brahme, R., O’Neill, Z., Lin, Y., Spears, M., Surana, A., Cerpa, A., Erickson, V., Kamthe, A., Eisenhower, B., Mezić, I. 2010 "Energy Performance Visualization and OccupancyBased Energy Management Systems for Buildings: Implementation and Testing at the University of California, Merced." Final Report to US Department Of Energy and California Energy Commission-Public Interest Energy Research, Lawrence Berkeley National Lab.

Brown, K., 2002, "Setting Enhanced Performance Targets for a New University Campus: Benchmarks vs. Energy Standards as a Reference", in Proceedings of ACEEE 2002 Summer Study on Energy Efficiency in Buildings: American Council for Energy Efficient Economy. 
Emmerich, S.J. and Persily, A.K., 2001, "State-of-the-art Review of CO2 Demand Controlled Ventilation Technology and Application", National Institute of Standards and Technology Technical Report, NISTIR 6729.

Erickson, V.L., Lin, Y., Kamthe, A., Brahme, R., Surana, A., Cerpa, A.E., Sohn, M.D., Narayanan, S., 2009, "Energy Efficient Building Environment Control Strategies Using Realtime Occupancy Measurements", in Proc. of ACM BuildSys 2009, First ACM Workshop on Embedded Sensing Systems for Energy-Efficiency in Buildings, Berkeley, Calif., Nov., 2009.

Flake, B.A. 1998. "Parameter Estimation and Optimal Supervisory Control of Chilled Water Plants", Doctoral Dissertation, University of Wisconsin-Madison.

Granderson, J., Piette, M.A., Ghatikar, G., Price, P., 2009, "Building Energy Information Systems: State of the Technology and User Case Studies", Lawrence Berkeley National Laboratory, Technical report LBNL-2899E.

Gillespie, K.L., Haves, P., Hitchcock, R.J., Deringer, J.J. and Kinney, K., 2007. "A Specifications Guide for Performance Monitoring Systems", Lawrence Berkeley National Laboratory, Pacific Gas and Electric Company, Deringer Group, QuEST, Mar. 23, 2007. http://cbs.lbl.gov/performancemonitoring/specifications/

Haves, P., Hencey, B., Borrellli, F., Elliott, J., Ma, Y., Coffey, B., Bengea, S. and Wetter, M. 2010. "Model Predictive Control of HVAC Systems: Implementation and Testing at the University of California, Merced.” Final Report to US DOE and CEC PIER, LBNL.

Henze, G. P., Kalz, D. E., Liu, S., Felsmann, C. 2005. "Experimental Analysis of Model-Based Predictive Optimal Control," HVAC\&R Research, Vol. 11, No. 2, pp. 189-213.

Mills, E. and Mathew, P, 2009, "Monitoring-Based Commissioning: Benchmarking Analysis of 24 UC/CSU/IOU Projects", Lawrence Berkeley National Laboratory.

Ma, Y., Borrelli, F., Hencey, B., Packard, A, Bortoff, S., 2009, "Model Predictive Control of Thermal Storage in Building Cooling Systems", Proc. of $48^{\text {th }}$ IEEE Conference on Decision and Control, China, pp. 392-397.

Ma, Y., Borrelli, F., Hencey, B., Coffey, B., Bengea, S., Packard, A., Wetter, M., Haves, P., 2010, "Model Predictive Control for the Operation of Building Cooling Systems", To appear in Proc. of IEEE American Control Conference, Baltimore, Maryland.

Mathew, P., E. Mills, N. Bourassa, M. Brook. 2008. "Action-Oriented Benchmarking: Using the CEUS Database to Benchmark Commercial Buildings in California." Energy Engineering Vol. 105, No. 5, pp. 6-19.

O'Neill, Narayanan, S., and Brahme, R. 2010, "Model Based Thermal Load Estimation in Buildings", to appear in SimBuild 2010.

Taylor Engineering, 2002; University of California, Merced Academic Building, Detailed Energy Analysis Report, Dec. 30, 2002.

Wetter, M. 2009. "Modelica-based Modeling and Simulation to Support Research and Development in Building Energy and Control Systems." J. Building Performance Simulation, 2(2):143-161. 